\title{
Systematic Review and Meta-Analysis of Randomized Controlled Trials of Fuke Qianjin Tablet
}

\author{
Pan Jie $\left(\mathbb{D}\right.$, Zuo Kai-Ni ${ }^{D}$, Wang Xiao-Mei $\mathbb{D}^{D}$, Zhang Meng-Pei $(\mathbb{D}$, Wang Zhi-Heng $(\mathbb{D}$, \\ Zhang Hao-Xiang $\mathbb{B}$, and Zhu Wen-Tao $\mathbb{D}$
}

School of Management, Beijing University of Chinese Medicine, Beijing, China

Correspondence should be addressed to Zhu Wen-Tao; zhuwt@bucm.edu.cn

Received 8 September 2020; Revised 9 December 2020; Accepted 15 January 2021; Published 17 February 2021

Academic Editor: Francesca Mancianti

Copyright (c) 2021 Pan Jie et al. This is an open access article distributed under the Creative Commons Attribution License, which permits unrestricted use, distribution, and reproduction in any medium, provided the original work is properly cited.

\begin{abstract}
Purpose. The aim of the research was to evaluate the efficacy and safety associated with Fuke Qianjin tablet combined with conventional therapy in the treatment of pelvic inflammatory diseases and associated complications (endometritis) using a metaanalysis approach. Patients and Methods. We searched 8 electronic databases up to December 31, 2019, including PubMed, the Cochrane Library, Embase, Web of Science, CNKI, WanFang, VIP, and SinoMed. Eligible studies were clinical trials of Fuke Qianjin tablet combined with conventional therapy used in the treatment of acute pelvic inflammatory disease, chronic pelvic inflammatory disease, and endometritis. The meta-analysis was performed using STATA15 software. Results. A total of 125 RCTs $(n=14,494)$ were shortlisted for the meta-analysis, which included 23 trials for acute pelvic inflammatory disease, 69 trials for chronic pelvic inflammatory disease, and 33 trials for endometritis. The overall analysis illustrated Fuke Qianjin tablet combined with conventional therapy was significantly more efficacious than conventional therapy alone across all types of antibiotics treatment for acute pelvic inflammatory disease $(\mathrm{OR}=5.57,95 \% \mathrm{CI} 4.09-7.58, Z=10.90, p=0.001)$, chronic pelvic inflammatory disease $(\mathrm{OR}=4.70,95 \% \mathrm{CI} 4.07-5.42, Z=21.21, p=0.001)$ and endometritis $(\mathrm{OR}=5.09,95 \% \mathrm{CI} 4.03-6.43 ; Z=13.63, p=0.001)$ in both primary endpoints and secondary endpoints. There is also a trend that Fuke Qianjin tablet combined with conventional therapy has lower adverse reaction rates than conventional therapy alone. Conclusion. Fuke Qianjin tablet combined with conventional therapy showed better clinical efficacy in the treatment of acute pelvic inflammatory disease, chronic pelvic inflammatory disease, and endometritis. There were no obvious drug-related adverse reactions. Fuke Qianjin tablet presented advantages in shortening the remission time of clinical symptoms, reducing the concentration of serum inflammatory factors, improving endometrial thickness, menstruation, and reducing relapse rate.
\end{abstract}

\section{Introduction}

Pelvic inflammatory disease (PID), one of the most common infections in women of reproductive age, could lead to endometritis, oophoritis, salpingitis, tuboovarian abscess, peritonitis, and other complications [1-4]. Studies have shown that about $25 \%$ of PID patients will have long-term sequelae, including annexitis, irregular menstruation, infertility, ectopic pregnancy, or chronic pelvic pain [5]. Moreover, compared with those without PID, patients with PID had a 1.864 times higher risk of developing preterm labor and a 2.121 times higher risk of developing ectopic pregnancy [6]. Women with PID also have a $6 \%-8 \%$ risk of infertility [5]. Endometritis or complications of PID will further reduce endometrial receptivity and increase the risk of infertility. The prevalence of chronic endometritis in the general population is about 19\% [7], and the prevalence of chronic endometritis in infertile patients is about 45\% [8]. Studies have shown that effective treatment of endometritis can improve patient reproductive health [9]. A research reported on PID health economics showed that young women are willing to spend 1-2 years of life to prevent PID and its related sequelae [10]. PID has severely affected female reproductive health and has also caused a heavy economic burden. In the United States, the medical cost in treating a PID case is about US\$3,200 [11], and the annual PID-related 
treatment cost on the US healthcare system adds to US $\$ 4.2$ billion [12]. The current treatment methods for PID are mainly antibiotic treatment and surgical treatment. According to the guidelines [13] issued by CDC in 2015, PID treatment programs must provide empirical and broadspectrum coverage of possible pathogens. However, longterm, repeated use of antibiotics may lead to increasing antibiotic resistance of related pathogens [14]. According to the 2018 World Health Organization (WHO) report, pathogens showed high rates of quinolone resistance, increasing azithromycin resistance, and emerging resistance to extended-spectrum cephalosporins [15]. On the other end, surgical treatment is only carried out when drug treatment is ineffective or emergency symptoms appear. Surgery presents risk of intrauterine iatrogenic damage, and so, surgical treatment measures should be carefully considered specific to the conditions of patients [16].

Fuke Qianjin tablet is a pure Chinese medicine preparation approved by the National Medical Products Administration. It is widely used clinically to treat acute pelvic inflammatory disease, chronic pelvic inflammatory disease, and endometritis. In recent years, many researchers have performed evaluation of the clinical application of Fuke Qianjin tablets [17], but further evaluation is deemed necessary. Therefore, this study aims to comprehensively evaluate the efficacy and safety of Fuke Qianjin tablets and provide a basis for its application as a treatment for pelvic inflammatory disease.

\section{Materials and Methods}

2.1. Literature Search. This study evaluated the clinical effectiveness and safety of Fuke Qianjin tablets in the treatment of acute pelvic inflammatory disease, chronic pelvic inflammatory disease, and endometritis. Synonyms of Fuke Qianjin tablets such as "Fuke Qianjin tablet," "Qianjin tablet," and "Qianjin" were used to search English databases. Chinese database search strategies and results are shown in Table 1. Additional, a manual search on chictr.org.cn (clinical trial database in China) was also done to identify potential unpublished studies.

2.2. Inclusion and Exclusion Criteria. Eligible studies should have met the following criteria: (1) randomized controlled trials (RCTs) of Fuke Qianjin tablets published in China or internationally; (2) patients diagnosed with acute pelvic inflammatory disease, chronic pelvic inflammatory disease, or endometritis; (3) the intervention measures of the experimental group are Fuke Qianjin tablets alone or combined with conventional treatment; and (4) the study reported one or more of the following outcome indicators: the main outcome indicators such as total effective rate and incidence of adverse reactions, and secondary outcome indicators such as clinical symptom relief time, endometrial thickness, serum inflammatory factor concentration, menstrual recovery, and relapse rate.

Exclusion criteria were (1) nonpharmacological treatment-related studies, such as acupuncture, physical therapy, and hot compresses; (2) studies with repeated publications; (3) studies with completely missing data; and (4) studies with unclear interventions, unclear description of efficacy evaluation criteria, or statistical errors.

2.3. Quality Assessment. Two authors independently evaluated the risk of bias of the included literature using the Cochrane Handbook which consists of seven domains [18]: random sequence generation, allocation concealment, blinding of participants and personnel, blinding of outcome assessments, incomplete outcome data, selective reporting, and other bias. For each domain, the risk of bias for each study was assessed according to three categories: low risk, high risk, or unclear risk. Disagreements encountered during the process were settled by a third author through discussion.

2.4. Data Extraction. Two independent reviewers extracted the following data from the included studies using a standardized data extraction form. Data are connected with study characteristics (first author's name, the sample size of each group, and publication year), patient characteristics (age and course of the disease), treatment measures, outcome measures, and literature quality evaluation information. Any disagreement between the two reviewers was subject to a discussion or further inquiry from the third researcher.

2.5. Statistical Analysis. All analyses were performed using STATA version 15.0. For dichotomous data, we calculated odds ratio (OR) with $95 \% \mathrm{CI}$; for continuous outcome data, we calculated the standard mean difference (SMD) with 95\% CI. In this case, the Cochrane Q statistic and $I^{2}$ statistics were conducted to test the heterogeneity across the included studies [19-22], in which $p$ value $<0.1$ or $I^{2}$ $>50 \%$ was deemed to represent substantial heterogeneity; then, the data were pooled using a random-effect model. On the contrary, the fixed-effect model is adopted. Publication bias was assessed by directly observing the symmetry of the inverted funnel chart, using the Harbord test and Egger's test [23-25]. In the case of significant publication bias, the trim and fill method [26] was used to correct the effect to verify the robustness of the metaanalysis results.

\section{Results}

3.1. Retrieval Results and Quality Assessment. All the retrieved literature $(n=1,434)$ were imported into the NoteExpress V3.2.0 version (NoteExpress, Beijing Aegean Sea Lezhi Technology Co., Ltd.). A 95\% accuracy algorithm was set to assess duplicates, and then, additional duplicate checks were performed manually. After screening with inclusion and exclusion criteria, a total of 125 separate studies were included for the final analysis (Figure 1), including 23 for acute pelvic inflammatory disease [27-49], 69 for chronic 
TABle 1: Chinese database retrieval strategy and results.

\begin{tabular}{|c|c|c|}
\hline Database & Search strategy & Outcome \\
\hline CNKI & $\begin{array}{c}\text { (SU }=(\text { 'Pelvic inflammatory disease'+'Chronic pelvic inflammatory disease'+' Pelvic inflammatory sequelae } \\
\text { '+")*('Fuke Qianjin Tablet ')) NOT TI=('rat') }\end{array}$ & 252 \\
\hline WanFang & $\begin{array}{c}\text { ((subject:(“Pelvic inflammatory disease"+"Chronic pelvic inflammatory disease"+"Pelvic inflammatory } \\
\text { sequelae"+"acute pelvic inflammatory disease")*("Fuke Qianjin Tablet")) not subject : ("rat"+"rabbit"+"Meta"))* } \\
\text { Date:-2019 }\end{array}$ & 387 \\
\hline VIP & $\begin{array}{l}(\mathrm{M}=\text { ("Pelvic inflammatory disease"+"Chronic pelvic inflammatory disease"+"Pelvic inflammatory } \\
\text { sequelae"+"acute pelvic inflammatory disease") AND (acute pelvic inflammatory disease "Fuke Qianjin Tablet") } \\
\text { ("Fuke Qianjin Tablet" Djangoorm: Intelligence AND("Pelvic inflammatory disease" Djangoorm: Intelligence OR }\end{array}$ & 214 \\
\hline SinoMed & $\begin{array}{l}\text { "Chronic pelvic inflammatory disease" Djangoorm: Intelligence OR "Pelvic inflammatory sequelae" Djangoorm: } \\
\text { Intelligence OR "acute pelvic inflammatory disease" Djangoorm: Intelligence)) NOT("rat" Djangoorm: Intelligence) }\end{array}$ & 278 \\
\hline CNKI & $\begin{array}{c}(\mathrm{SU}=(\text { 'endometritis'+'chronic endometritis'+'Acute endometritis'+'puerperal endometritis'+'puerperium } \\
\text { endometritis')*(' Fuke Qianjin Tablet ')) NOT TI=('rat') }\end{array}$ & 78 \\
\hline WanFang & $\begin{array}{l}\text { ((subject: ("endometritis"+"chronic endometritis"+"Acute endometritis"+"puerperal endometritis"+"puerperium } \\
\text { endometritis")* ("Fuke Qianjin Tablet")) not subject : ("rat"+"rabbit"+"Meta"))*Date:-2019 }\end{array}$ & 87 \\
\hline VIP & (M=(“endometritis"+"chronic endometritis"+"Acute endometritis") AND (“Fuke Qianjin Tablet") & 74 \\
\hline SinoMed & $\begin{array}{c}\text { (“Fuke Qianjin Tablet” Djangoorm: Intelligence AND ("endometritis" Djangoorm: Intelligence OR "chronic } \\
\text { endometritis" Djangoorm: Intelligence OR “Acute endometritis” Djangoorm: Intelligence OR "puerperal } \\
\text { endometritis" Djangoorm: Intelligence OR "puerperium endometritis" Djangoorm: Intelligence)) NOT("rat" } \\
\text { Djangoorm: Intelligence OR “meta” Djangoorm: Intelligence) }\end{array}$ & 54 \\
\hline
\end{tabular}

pelvic inflammatory disease [50-118], and 33 for endometritis [119-151]. The full list of studies is illustrated in Tables 1-3.

\subsection{Meta-Analysis of Fuke Qianjin Tablet in the Treatment of Acute Pelvic Inflammatory}

\subsubsection{The Main Outcome}

(1) Total of Effective Rate. There were 23 articles [27-49] about Fuke Qianjin tablets combined with antibiotics in the treatment of acute pelvic inflammatory disease. The results showed that Fuke Qianjin tablets combined with antibiotics led to significantly better clinical efficacy compared to antibiotics alone $(\mathrm{OR}=5.57,95 \% \mathrm{CI} 4.09-7.58, Z=10.90$, $p=0.001)$. The results of the subgroup analysis showed that the efficacy of FKQJP combined with antibiotics was not affected by antibiotic types (Table 4 and Figure 2).

(2) Adverse Reaction. A total of 15 studies reported outcome of adverse reactions, in which 10 studies reported the specific data of 90 cases of adverse reactions. The difference of the incidence of the adverse reaction between the two groups was not statistically significant $(\mathrm{OR}=0.71,95 \%$ CI $0.45-1.10$, $Z=1.55, p=0.121) .5$ studies reported no adverse events or mild adverse reactions which improved spontaneously (Table 4 and Figure 3).

3.2.2. Secondary Outcomes. Comparisons in secondary outcomes were also made. Similar to the primary outcome, Fuke Qianjin tablets combined with antibiotics had better efficacy profiles in shortening the remission time of clinical symptoms and reducing serum inflammatory factor concentrations when compared with antibiotics alone, as shown in Table 5.
3.2.3. Publication Bias. The publication bias test was performed on the total effective rate and incidence of adverse reactions, and the inverted funnel diagram (Figures 4(a) and 4(b)) was drawn, respectively. The results of the Harbord test showed that there were significant publication bias and small sample effect in the outcome of the total effective rate ( $t=5.95, p=0.001)$, and there were not significant publication bias and small sample effect in the outcome of the incidence of adverse reactions $(t=-0.57, p=0.583)$.

The sensitivity analysis was carried out by using the trim and filled method. The point estimated value and 95\% confidence interval of the summary effect OR before and after the implementation of the trim and fill method was 5.57 $[4.09,7.58]$ and $4.25[3.18,5.68]$, respectively. The differences between two of groups were still statistically significant $(Z=9.765, p=0.001)$. The iterative results show that 10 additional studies are needed to further enhance the stability of the research results.

\subsection{Meta-Analysis of Fuke Qianjin Tablet in the Treatment of Chronic Pelvic Inflammatory}

\subsubsection{The Main Outcome}

(1) Total of Effective Rate. There were 69 articles about Fuke Qianjin tablets combined with antibiotics in the treatment of chronic pelvic inflammatory disease [50-118]. The results showed that Fuke Qianjin tablets combined with antibiotics had better clinical efficacy $(\mathrm{OR}=4.70,95 \%$ CI 4.07-5.42, $Z=21.21, p=0.001)$ than antibiotics alone. The results of the subgroup analysis showed that the efficacy of Fuke Qianjin tablets combined with antibiotics was not affected by antibiotic types (Table 4 and Figure 5).

(2) Adverse Reaction. A total of 30 studies reported adverse reactions to the treatment of chronic pelvic inflammation. Among them, 26 studies reported specific data. The fixed- 


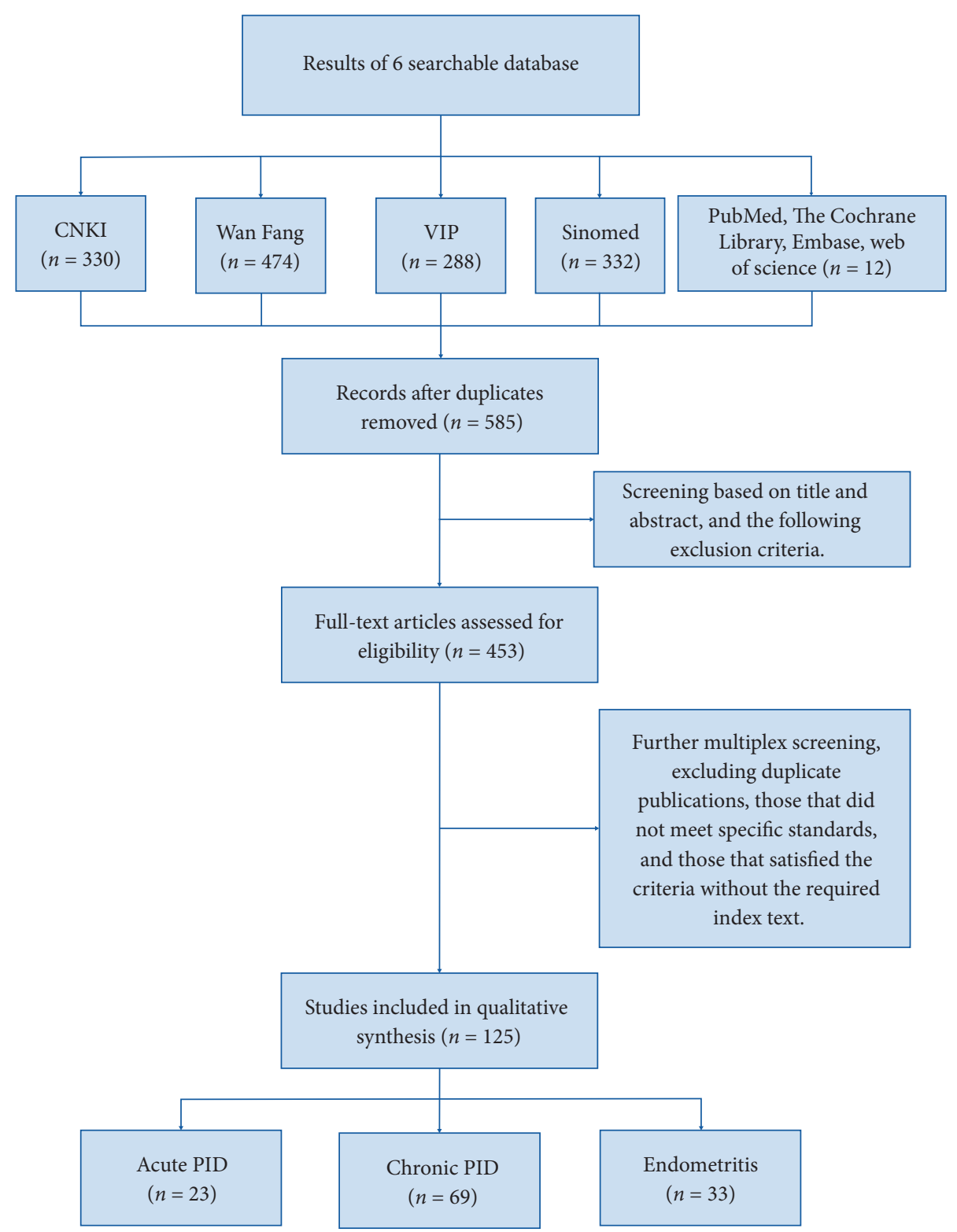

FIgUre 1: Study selection steps.

effects model analysis showed that compared with the control group, the incidence of adverse reactions in the combined group of Fuke Qianjin tablets was lower $(\mathrm{OR}=0.60,95 \% \mathrm{CI} 0.46-0.80, Z=3.55, p=0.001)$. (Table 4 and Figure 6).

3.3.2. Secondary Outcomes. Comparisons in secondary outcomes were also made. Similar to the primary outcome, Fuke Qianjin tablets combined with antibiotics had better efficacy profiles in shortening the remission time of clinical symptoms and reducing serum inflammatory factor concentrations when compared with antibiotics alone, as shown in Table 5.

3.3.3. Publication Bias. Perform the publication bias test on total effective rate and the incidence of adverse reactions, and the inverted funnel diagram (Figures 7(a) and 7(b)) was drawn, respectively. The inverted funnel plots of the 2 outcomes were substantially symmetric. The results of the Harbord test showed that there were no significant publication bias and small sample effect in the total effective rate $(t=0.11, p=0.912)$ and adverse reaction rate $(t=0.96$, $p=0.349)$.

\subsection{Meta-Analysis of Fuke Qianjin Tablet in the Treatment of Endometritis}

\subsubsection{The Main Outcome}

(1) Total of Effective Rate. There were 33 studies about Fuke Qianjin tablets combined with conventional treatment of endometritis, and 30 studies reported the total effective 
TABLE 2: Characteristics of the studies included in this meta-analysis.

\begin{tabular}{|c|c|c|c|c|c|}
\hline Author & $\mathrm{Na} / \mathrm{Nb}$ & Course & Experimental & Control & Outcome \\
\hline \multicolumn{6}{|c|}{ Acute pelvic inflammatory } \\
\hline CCJ $(2018)[27]$ & $35 / 35$ & 15 & FKQJP + C & Penicillin & (1) (3) \\
\hline YML (2013) [28] & $24 / 23$ & 12 & $\mathrm{FKQJP}+\mathrm{C}$ & Penicillin & (1) \\
\hline ZJ (2017) [29] & $48 / 48$ & 15 & FKQJP + C & Penicillin & (1) (3) \\
\hline DY (2014) [30] & $68 / 68$ & $10 \sim 15$ & FKQJP + C & Penicillin & (1) (2) (3) (4) \\
\hline SL (2015) [31] & $50 / 50$ & 10 & FKQJP + C & Penicillin & (1) (2) (4) \\
\hline TSQ (2019) [32] & $50 / 50$ & 10 & $\mathrm{FKQJP}+\mathrm{C}$ & Penicillin & (1) \\
\hline XQX (2014) [33] & $39 / 39$ & 15 & FKQJP + C & Penicillin + metronidazole & (1) (2) (3) \\
\hline YFY (2013) [34] & $67 / 68$ & $10 \sim 15$ & $\mathrm{FKQJP}+\mathrm{C}$ & Penicillin + metronidazole & (1) (3) \\
\hline RS (2012) [35] & $36 / 36$ & 10 & FKQJP + C & Penicillin + metronidazole & (1) (2) \\
\hline SFM (2015) [36] & $66 / 66$ & 10 & $\mathrm{FKQJP}+\mathrm{C}$ & Penicillin + metronidazole & (1) (2) \\
\hline ZY (2013) [37] & $43 / 43$ & 10 & FKQJP + C & Penicillin + metronidazole & (1) (4) \\
\hline FXH (2018) [38] & $39 / 39$ & 14 & FKQJP + C & Penicillin + metronidazole & (1) (2) (3) (4) \\
\hline SYQ (2015) [39] & $\begin{array}{l}110 / \\
110\end{array}$ & 15 & $\mathrm{FKQJP}+\mathrm{C}$ & Penicillin + metronidazole & (1) (3) \\
\hline ZHY (2012) [40] & $60 / 60$ & 10 & $\mathrm{FKQJP}+\mathrm{C}$ & Roxithromycin & (1) (2) \\
\hline $\mathrm{ZYZ}(2012)[41]$ & $30 / 30$ & 12 & $\mathrm{FKQJP}+\mathrm{C}$ & Roxithromycin & (1) (2) \\
\hline YCQ (2017) [42] & $60 / 60$ & $7 \sim 14$ & $\mathrm{FKQJP}+\mathrm{C}$ & Moxifloxacin & (1) (2) \\
\hline LWR (2013) [43] & $43 / 43$ & 10 & $\mathrm{FKQJP}+\mathrm{C}$ & Levofloxacin hydrochloride & (1) (3) (4) \\
\hline WGJ (2011) [44] & $50 / 50$ & 14 & FKQJP + C & Ceftriaxone + tinidazole & (1) (2) \\
\hline HRM (2013) [45] & $60 / 60$ & $7 \sim 15$ & FKQJP + C & Ceftriaxone + tinidazole & (1) (2) \\
\hline GSY (2012) [46] & $30 / 30$ & 10 & FKQJP + C & Levofloxacin lactate + metronidazole & (1) (2) \\
\hline ZYH (2016) [47] & $40 / 40$ & $7 \sim 14$ & FKQJP + C & Gatifloxacin + metronidazole & (1) (2) \\
\hline ZNN (2012) [48] & $30 / 30$ & 12 & FKQJP + C & Metronidazole & (1) (2) \\
\hline LJ (2014) [49] & $36 / 36$ & 14 & FKQJP + C & Metronidazole & (1) (4) \\
\hline \multicolumn{6}{|c|}{ Chronic pelvic inflammatory } \\
\hline LJX2 (2014) [50] & $40 / 40$ & 42 & FKQJP & $\beta$-Lactam + nitroimidazole & (1) \\
\hline XZJ (2007) [51] & $\begin{array}{c}102 / \\
102\end{array}$ & $45 / 21$ & FKQJP & $\beta$-Lactam + nitroimidazole & (1) \\
\hline CXC (2019) [52] & $64 / 64$ & 21 & $\mathrm{FKQJP}+\mathrm{C}$ & $\beta$-Lactam & (1) (4) \\
\hline DJL (2014) [53] & $62 / 62$ & 21 & $\mathrm{FKQJP}+\mathrm{C}$ & $\beta$-Lactam & (1) (2) \\
\hline PGZ (2016) [54] & $40 / 40$ & 14 & FKQJP + C & $\beta$-Lactam & (1) (2) (3) \\
\hline SXL (2015) [55] & $32 / 32$ & 21 & FKQJP + C & $\beta$-Lactam & (1) (3) \\
\hline WQF (2013) [56] & $35 / 35$ & 20 & FKQJP + C & $\beta$-Lactam & (1) \\
\hline ZXJ (2015) [57] & $30 / 30$ & 14 & FKQJP + C & $\beta$-Lactam & (1) (2) \\
\hline ZMY (2017) [58] & $50 / 50$ & 14 & FKQJP + C & $\beta$-Lactam & (1) (2) \\
\hline LJX1 (2014) [50] & $40 / 40$ & 42 & FKQJP + C & $\beta$-Lactam + nitroimidazole & (1) \\
\hline LXX (2019) [59] & $43 / 43$ & 15 & FKQJP + C & $\beta$-Lactam + nitroimidazole & (1) \\
\hline LH (2019) [60] & $52 / 52$ & 14 & FKQJP + C & $\beta$-Lactam + nitroimidazole & (1) (2) \\
\hline PJQ (2019) [61] & $75 / 75$ & 15 & FKQJP + C & $\beta$-Lactam + nitroimidazole & (1) \\
\hline SWA (2014) [62] & $36 / 36$ & 28 & FKQJP + C & $\beta$-Lactam + nitroimidazole & (1) \\
\hline SQB2 (2012) [63] & $50 / 50$ & 28 & $\mathrm{FKQJP}+\mathrm{C}$ & $\beta$-Lactam + nitroimidazole & (1) (2) \\
\hline $\mathrm{ZY}(2014)[64]$ & $54 / 54$ & 28 & FKQJP + C & $\beta$-Lactam + nitroimidazole & (1) (2) \\
\hline SQB1 (2012) [63] & $50 / 50$ & 28 & $\begin{array}{c}\text { FKQJP }+\beta- \\
\text { lactam + nitroimidazole }\end{array}$ & FKQJP & (1) (2) \\
\hline SPF (2015) [65] & $75 / 75$ & 14 & $\begin{array}{c}\text { FKQJP }+\beta- \\
\text { lactam }+ \text { nitroimidazole }\end{array}$ & FKQJP & (1) (2) \\
\hline CF (2018) [66] & $60 / 60$ & 42 & FKQJP $+\mathrm{C}$ & Macrolide & (1) (2) \\
\hline DLL (2017) [67] & $81 / 81$ & 42 & $\mathrm{FKQJP}+\mathrm{C}$ & Macrolide & (1) (2) \\
\hline FJY (2010) [68] & $63 / 63$ & 15 & FKQJP + C & Macrolide & (1) (2) \\
\hline FP (2013) [69] & $62 / 62$ & 14 & $\mathrm{FKQJP}+\mathrm{C}$ & Macrolide & (1) \\
\hline KY (2018) [70] & $100 / 86$ & 14 & FKQJP + C & Macrolide & (1) (3) \\
\hline LMY (2016) [71] & $50 / 50$ & 14 & FKQJP + C & Macrolide & (1) \\
\hline LYK (2016) [72] & $30 / 30$ & 15 & FKQJP + C & Macrolide & (1) (2) \\
\hline LongH (2011) [73] & $90 / 90$ & 28 & FKQJP + C & Macrolide & (1) \\
\hline RDQ (2019) [74] & $40 / 40$ & 21 & FKQJP + C & Macrolide & (1) \\
\hline ShaoXL (2012) [75] & $87 / 86$ & 15 & FKQJP + C & Macrolide & (1) \\
\hline TXG (2015) [76] & $90 / 90$ & 15 & FKQJP + C & Macrolide & (1) (2) \\
\hline WJP (2013) [77] & $52 / 48$ & 51 & FKQJP + C & Macrolide & (1) \\
\hline YM (2017) [78] & $50 / 50$ & 51 & FKQJP + C & Macrolide & (1) \\
\hline YQ (2014) [79] & $67 / 67$ & 15 & FKQJP + C & Macrolide & (1) (2) \\
\hline ZYL (2017) [80] & $40 / 40$ & 20 & FKQJP + C & Macrolide & (1) (2) \\
\hline
\end{tabular}


TABle 2: Continued.

\begin{tabular}{|c|c|c|c|c|c|}
\hline Author & $\mathrm{Na} / \mathrm{Nb}$ & Course & Experimental & Control & Outcome \\
\hline DLH (2019) [81] & $45 / 45$ & 30 & FKQJP + C & Quinolone & (1) \\
\hline HJY (2019) [82] & $31 / 31$ & 30 & FKQJP + C & Quinolone & (1) (4) \\
\hline LJ (2019) [83] & $22 / 22$ & 14 & $\mathrm{FKQJP}+\mathrm{C}$ & Quinolone & (1) (2) \\
\hline SJL (2016) [84] & $53 / 53$ & 30 & FKQJP + C & Quinolone & (1) \\
\hline WJ (2018) [85] & $40 / 40$ & 14 & $\mathrm{FKQJP}+\mathrm{C}$ & Quinolone & (1) \\
\hline ZL (2015) [86] & $40 / 40$ & 21 & FKQJP + C & Quinolone & (1) \\
\hline ZT (2018) [87] & $\begin{array}{c}160 / \\
160\end{array}$ & 21 & $\mathrm{FKQJP}+\mathrm{C}$ & Quinolone & (1) (4) \\
\hline ZhaoJ (2017) [88] & $55 / 55$ & 28 & $\mathrm{FKQJP}+\mathrm{C}$ & Quinolone & (1) (4) \\
\hline ZengJ (2016) [89] & $40 / 40$ & 14 & FKQJP + C & Quinolone + nitroimidazole & (1) (2) \\
\hline CXF (2018) [90] & $58 / 58$ & 42 & $\mathrm{FKQJP}+\mathrm{C}$ & Quinolone + nitroimidazole & (1) (3) \\
\hline $\operatorname{DCM}(2016)[91]$ & $47 / 47$ & 28 & $\mathrm{FKQJP}+\mathrm{C}$ & Quinolone + nitroimidazole & (1) (3) \\
\hline DQY (2015) [92] & $25 / 25$ & 28 & $\mathrm{FKQJP}+\mathrm{C}$ & Quinolone + nitroimidazole & (1) (2) \\
\hline HSX (2019) [93] & $65 / 65$ & 42 & $\mathrm{FKQJP}+\mathrm{C}$ & Quinolone + nitroimidazole & (1) (2) \\
\hline HHJ (2019) [94] & $60 / 60$ & 28 & $\mathrm{FKQJP}+\mathrm{C}$ & Quinolone + nitroimidazole & (1) \\
\hline HHM (2019) [95] & $63 / 63$ & 28 & $\mathrm{FKQJP}+\mathrm{C}$ & Quinolone + nitroimidazole & (1) (2) (3) (4) \\
\hline HYL (2015) [96] & $60 / 60$ & 32 & $\mathrm{FKQJP}+\mathrm{C}$ & Quinolone + nitroimidazole & (1) (2) \\
\hline LML (2019) [97] & $29 / 29$ & 14 & $\mathrm{FKQJP}+\mathrm{C}$ & Quinolone + nitroimidazole & (1) (3) \\
\hline LHR (2013) [98] & $45 / 41$ & 28 & $\mathrm{FKQJP}+\mathrm{C}$ & Quinolone + nitroimidazole & (1) (2) (3) \\
\hline LXL (2018) [99] & $41 / 41$ & 14 & FKQJP + C & Quinolone + nitroimidazole & (1) (4) \\
\hline LSY (2018) [100] & $\begin{array}{c}587 / \\
588\end{array}$ & 28 & $\mathrm{FKQJP}+\mathrm{C}$ & Quinolone + nitroimidazole & (1) (3) (4) \\
\hline LYZ (2012) [101] & $35 / 35$ & 28 & $\mathrm{FKQJP}+\mathrm{C}$ & Quinolone + nitroimidazole & (1) (3) \\
\hline MYQ (2019) [102] & $30 / 30$ & 28 & $\mathrm{FKQJP}+\mathrm{C}$ & Quinolone + nitroimidazole & (1) \\
\hline $\mathrm{RC}(2016)[103]$ & $60 / 60$ & 14 & $\mathrm{FKQJP}+\mathrm{C}$ & Quinolone + nitroimidazole & (1) \\
\hline RL (2017) [104] & $43 / 43$ & 28 & $\mathrm{FKQJP}+\mathrm{C}$ & Quinolone + nitroimidazole & (1) (2) \\
\hline $\begin{array}{l}\text { SNY·HSY (2016) } \\
{[105]}\end{array}$ & $40 / 40$ & 20 & $\mathrm{FKQJP}+\mathrm{C}$ & Quinolone + nitroimidazole & (1) (2) \\
\hline SQJ (2012) [106] & $30 / 30$ & 14 & $\mathrm{FKQJP}+\mathrm{C}$ & Quinolone + nitroimidazole & (1) (2) \\
\hline YLH (2012) [107] & $42 / 42$ & 28 & $\mathrm{FKQJP}+\mathrm{C}$ & Quinolone + nitroimidazole & (1) (3) (4) \\
\hline WDD (2017) [108] & $35 / 35$ & 28 & $\mathrm{FKQJP}+\mathrm{C}$ & Quinolone + nitroimidazole & (1) \\
\hline WHL (2011) [109] & $90 / 90$ & 28 & $\mathrm{FKQJP}+\mathrm{C}$ & Quinolone + nitroimidazole & (1) (3) \\
\hline WLY (2019) [110] & $50 / 50$ & 30 & $\mathrm{FKQJP}+\mathrm{C}$ & Quinolone + nitroimidazole & (1) (2) \\
\hline WSY (2011) [111] & $50 / 50$ & 90 & $\mathrm{FKQJP}+\mathrm{C}$ & Quinolone + nitroimidazole & (1) \\
\hline WY (2018) [112] & $36 / 36$ & $28 \sim 42$ & $\mathrm{FKQJP}+\mathrm{C}$ & Quinolone + nitroimidazole & (1) \\
\hline WDM (2019) [113] & $48 / 48$ & 28 & FKQJP + C & Quinolone + nitroimidazole & (1) (2) \\
\hline XW (2015) [114] & $65 / 63$ & 28 & $\mathrm{FKQJP}+\mathrm{C}$ & Quinolone + nitroimidazole & (1) (3) \\
\hline XRQ (2018) [115] & $37 / 37$ & 28 & $\mathrm{FKQJP}+\mathrm{C}$ & Quinolone + nitroimidazole & (1) (3) \\
\hline YDM (2015) [116] & $30 / 30$ & 28 & $\mathrm{FKQJP}+\mathrm{C}$ & Quinolone + nitroimidazole & (1) (2) \\
\hline YXH (2016) [117] & $53 / 53$ & 42 & FKQJP + C & Quinolone + nitroimidazole & (1) (2) \\
\hline ZhangJ (2017) [118] & $35 / 35$ & 14 & $\mathrm{FKQJP}+\mathrm{C}$ & Quinolone + nitroimidazole & (1) (2) \\
\hline \multicolumn{6}{|l|}{ Endometritis } \\
\hline SFF (2017) [119] & $44 / 44$ & 45 & $\mathrm{FKQJP}+\mathrm{C}$ & Cefoxitin + doxycycline & (4) \\
\hline SCP (2019) [120] & $46 / 46$ & 45 & $\mathrm{FKQJP}+\mathrm{C}$ & Cefoxitin + doxycycline & (1) \\
\hline DYY (2019) [121] & $\begin{array}{l}174 / \\
174\end{array}$ & 45 & FKQJP + C & Cefoxitin + doxycycline & (1) (3) (4) \\
\hline LY (2019) [122] & $34 / 33$ & 45 & $\mathrm{FKQJP}+\mathrm{C}$ & Cefoxitin + doxycycline & (3) (4) \\
\hline HHF (2017) [123] & $60 / 60$ & 21 & $\mathrm{FKQJP}+\mathrm{C}$ & Levofloxacin & (1) (2) (3) (4) \\
\hline WJF (2019) [124] & $60 / 60$ & 21 & FKQJP + C & Levofloxacin & (1) (3) (4) \\
\hline MTX (2016) [125] & $40 / 40$ & $14 / 42$ & FKQJP + metronidazole & Metronidazole + medroxyprogesterone & (1) (3) (5) \\
\hline SXW (2018) [126] & $45 / 45$ & 42 & FKQJP + metronidazole & Metronidazole + medroxyprogesterone & (1) (2) (4) \\
\hline $\mathrm{ZHH}(2018)[127]$ & $52 / 52$ & 42 & FKQJP + metronidazole & Metronidazole + medroxyprogesterone & (1) (3) (5) \\
\hline ZL (2019) [128] & $34 / 34$ & 30 & FKQJP + metronidazole & Metronidazole + medroxyprogesterone & (1) (3) (4) \\
\hline DCL (2017) [129] & $20 / 20$ & 42 & FKQJP + metronidazole & Metronidazole + medroxyprogesterone & (1) \\
\hline DFY (2017) [130] & $80 / 80$ & 42 & FKQJP + metronidazole & Metronidazole + medroxyprogesterone & (1) (3) (5) \\
\hline GY (2015) [131] & $78 / 78$ & $14 / 42$ & FKQJP + metronidazole & Metronidazole + medroxyprogesterone & (1) (4) \\
\hline LWP (2019) [132] & $39 / 39$ & 30 & FKQJP + metronidazole & Metronidazole + medroxyprogesterone & (1) \\
\hline MYJ (2018) [133] & $42 / 42$ & 42 & FKQJP + metronidazole & Metronidazole + medroxyprogesterone & (1) \\
\hline DFL (2017) [134] & $41 / 41$ & 42 & FKQJP + metronidazole & Metronidazole + medroxyprogesterone & (1) (3) (4) \\
\hline HXY (2018) [135] & $50 / 50$ & $14 / 42$ & FKQJP + metronidazole & Metronidazole + medroxyprogesterone & (1) (4) \\
\hline ZQ (2019) [136] & $40 / 40$ & 42 & FKQJP + metronidazole & Metronidazole + norethisterone & (1) (3) (4) \\
\hline LXZ (2017) [137] & $64 / 64$ & 14 & FKQJP + C & Metronidazole + medroxyprogesterone & (1) (2) (3) \\
\hline
\end{tabular}


TABLE 2: Continued.

\begin{tabular}{|c|c|c|c|c|c|}
\hline Author & $\mathrm{Na} / \mathrm{Nb}$ & Course & Experimental & Control & Outcome \\
\hline CXH (2017) [138] & $30 / 30$ & 60 & $\mathrm{FKQJP}+\mathrm{C}$ & Metronidazole + medroxyprogesterone & (1) \\
\hline FCF (2016) [139] & $90 / 90$ & $14 / 42$ & $\mathrm{FKQJP}+\mathrm{C}$ & Metronidazole + medroxyprogesterone & (1) (3) (4) \\
\hline LBY (2019) [140] & $30 / 30$ & $14 / 42$ & $\mathrm{FKQJP}+\mathrm{C}$ & Metronidazole + medroxyprogesterone & (1) (3) (4) \\
\hline LSQ (2017) [141] & $45 / 44$ & 42 & $\mathrm{FKQJP}+\mathrm{C}$ & Metronidazole + medroxyprogesterone & (1) (2) (3) (4) \\
\hline YL (2018) [142] & $40 / 40$ & $14 / 42$ & $\mathrm{FKQJP}+\mathrm{C}$ & Metronidazole + medroxyprogesterone & (1) (4) \\
\hline ZQ (2016) [143] & $\begin{array}{c}156 / \\
156\end{array}$ & $14 / 60$ & FKQJP + C & Metronidazole + medroxyprogesterone & (1) (4) (5) \\
\hline ZCY (2018) [144] & $14 / 14$ & 42 & $\mathrm{FKQJP}+\mathrm{C}$ & Metronidazole + medroxyprogesterone & (1) (4) \\
\hline ZGB (2017) [145] & $41 / 41$ & 42 & FKQJP + C & Metronidazole + medroxyprogesterone & (1) \\
\hline GLJNT (2017) [146] & $90 / 90$ & 42 & $\mathrm{FKQJP}+\mathrm{C}$ & Metronidazole + norethisterone & (1) (3) \\
\hline CJH (2016) [147] & $40 / 40$ & 42 & FKQJP + C & Metronidazole + norethisterone & (1) (3) \\
\hline AYGL (2018) [148] & $40 / 40$ & 42 & $\mathrm{FKQJP}+\mathrm{C}$ & Metronidazole + norethisterone & (3) (4) \\
\hline SXX (2018) [149] & $70 / 70$ & 42 & $\mathrm{FKQJP}+\mathrm{C}$ & $\begin{array}{c}\text { Metronidazole }+ \text { norethisterone }+ \text { vaginal } \\
\text { douche }\end{array}$ & (1) (3) (4) \\
\hline XCY (2015) [150] & $75 / 75$ & 21 & $\mathrm{FKQJP}+\mathrm{C}$ & $\begin{array}{c}\text { Metronidazole }+ \text { norethisterone }+ \text { vaginal } \\
\text { douche }\end{array}$ & (1) (3) (4) \\
\hline HF (2016) [151] & $75 / 75$ & 21 & $\mathrm{FKQJP}+\mathrm{C}$ & $\begin{array}{c}\text { Metronidazole }+ \text { norethisterone }+ \text { vaginal } \\
\text { douche }\end{array}$ & (1) (4) \\
\hline
\end{tabular}

Note. (1), total effective rate; (2), incidence of adverse reaction; (3), the time of clinical symptom remission/disappearance; (4), serum inflammatory factor concentration. FKQJP, Fuke Qianjin tablet; C, control group regimens.

TABLE 3: Risk of bias evaluation of included literature.

\begin{tabular}{|c|c|c|c|c|c|c|c|}
\hline Study ID & $\begin{array}{c}\text { Random } \\
\text { sequence } \\
\text { generation } \\
\text { (selection bias) }\end{array}$ & $\begin{array}{c}\text { Allocation } \\
\text { concealment } \\
\text { (selection bias) }\end{array}$ & $\begin{array}{c}\text { Blinding of } \\
\text { participants and } \\
\text { personnel } \\
\text { (performance bias) }\end{array}$ & $\begin{array}{c}\text { Blinding of } \\
\text { outcome } \\
\text { assessment } \\
\text { (detection bias) }\end{array}$ & $\begin{array}{c}\text { Incomplete } \\
\text { outcome data } \\
\text { (attrition bias) }\end{array}$ & $\begin{array}{l}\text { Selective } \\
\text { reporting } \\
\text { (reporting } \\
\text { bias) }\end{array}$ & $\begin{array}{c}\text { Other } \\
\text { bias }\end{array}$ \\
\hline \multicolumn{8}{|c|}{ Acute pelvic inflammatory } \\
\hline $\begin{array}{l}\text { CCJ (2018) } \\
{[27]}\end{array}$ & $\mathrm{U}$ & $\mathrm{U}$ & $\mathrm{U}$ & $\mathrm{U}$ & $\mathrm{L}$ & $\mathrm{L}$ & $\mathrm{U}$ \\
\hline $\begin{array}{l}\text { YML (2013) } \\
\text { [28] }\end{array}$ & $\mathrm{U}$ & $\mathrm{U}$ & $\mathrm{U}$ & $\mathrm{U}$ & $\mathrm{L}$ & $\mathrm{L}$ & $\mathrm{U}$ \\
\hline $\begin{array}{l}\text { ZJ (2017) } \\
{[29]}\end{array}$ & $\mathrm{L}$ & $\mathrm{U}$ & $\mathrm{U}$ & $\mathrm{U}$ & $\mathrm{L}$ & $\mathrm{L}$ & $\mathrm{U}$ \\
\hline $\begin{array}{l}\text { DY (2014) } \\
\text { [30] }\end{array}$ & $\mathrm{U}$ & $\mathrm{U}$ & $\mathrm{U}$ & $\mathrm{U}$ & $\mathrm{L}$ & $\mathrm{L}$ & $\mathrm{U}$ \\
\hline $\begin{array}{l}\text { SL (2015) } \\
\text { [31] }\end{array}$ & $\mathrm{U}$ & $\mathrm{U}$ & $\mathrm{U}$ & $\mathrm{U}$ & $\mathrm{L}$ & $\mathrm{L}$ & $\mathrm{U}$ \\
\hline $\begin{array}{l}\text { TSQ (2019) } \\
\text { [32] }\end{array}$ & $\mathrm{U}$ & $\mathrm{U}$ & $\mathrm{U}$ & $\mathrm{U}$ & $\mathrm{L}$ & $\mathrm{L}$ & $\mathrm{U}$ \\
\hline $\begin{array}{l}\text { XQX (2014) } \\
{[33]}\end{array}$ & $\mathrm{U}$ & $\mathrm{U}$ & $\mathrm{U}$ & $\mathrm{U}$ & $\mathrm{L}$ & $\mathrm{H}$ & $\mathrm{U}$ \\
\hline $\begin{array}{l}\text { YFY (2013) } \\
\text { [34] }\end{array}$ & $\mathrm{U}$ & $\mathrm{U}$ & $\mathrm{U}$ & $\mathrm{U}$ & $\mathrm{L}$ & $\mathrm{L}$ & $\mathrm{U}$ \\
\hline $\begin{array}{l}\text { RS (2012) } \\
{[35]}\end{array}$ & $\mathrm{U}$ & $\mathrm{U}$ & $\mathrm{U}$ & $\mathrm{U}$ & $\mathrm{L}$ & $\mathrm{L}$ & $\mathrm{U}$ \\
\hline $\begin{array}{l}\text { SFM (2015) } \\
{[36]}\end{array}$ & U & $\mathrm{U}$ & $\mathrm{U}$ & $\mathrm{U}$ & $\mathrm{L}$ & $\mathrm{L}$ & $\mathrm{U}$ \\
\hline $\begin{array}{l}\text { ZY (2013) } \\
{[37]}\end{array}$ & U & $\mathrm{U}$ & $\mathrm{U}$ & $\mathrm{U}$ & $\mathrm{L}$ & $\mathrm{L}$ & $\mathrm{U}$ \\
\hline $\begin{array}{l}\text { FXH (2018) } \\
{[38]}\end{array}$ & $\mathrm{L}$ & $\mathrm{U}$ & $\mathrm{U}$ & $\mathrm{U}$ & $\mathrm{L}$ & $\mathrm{L}$ & $\mathrm{U}$ \\
\hline $\begin{array}{l}\text { SYQ (2015) } \\
\text { [39] }\end{array}$ & $\mathrm{U}$ & $\mathrm{U}$ & $\mathrm{U}$ & $\mathrm{U}$ & $\mathrm{L}$ & $\mathrm{L}$ & $\mathrm{U}$ \\
\hline $\begin{array}{l}\text { ZHY (2012) } \\
{[40]}\end{array}$ & $\mathrm{U}$ & $\mathrm{U}$ & $\mathrm{U}$ & $\mathrm{U}$ & $\mathrm{L}$ & $\mathrm{L}$ & $\mathrm{U}$ \\
\hline $\begin{array}{l}\text { ZYZ (2012) } \\
\text { [41] }\end{array}$ & $\mathrm{U}$ & $\mathrm{U}$ & $\mathrm{U}$ & $\mathrm{U}$ & $\mathrm{L}$ & $\mathrm{L}$ & $\mathrm{U}$ \\
\hline
\end{tabular}


TABle 3: Continued.

\begin{tabular}{|c|c|c|c|c|c|c|c|}
\hline Study ID & $\begin{array}{c}\text { Random } \\
\text { sequence } \\
\text { generation } \\
\text { (selection bias) }\end{array}$ & $\begin{array}{c}\text { Allocation } \\
\text { concealment } \\
\text { (selection bias) }\end{array}$ & $\begin{array}{c}\text { Blinding of } \\
\text { participants and } \\
\text { personnel } \\
\text { (performance bias) }\end{array}$ & $\begin{array}{c}\text { Blinding of } \\
\text { outcome } \\
\text { assessment } \\
\text { (detection bias) }\end{array}$ & $\begin{array}{c}\text { Incomplete } \\
\text { outcome data } \\
\text { (attrition bias) }\end{array}$ & $\begin{array}{c}\text { Selective } \\
\text { reporting } \\
\text { (reporting } \\
\text { bias) }\end{array}$ & $\begin{array}{c}\text { Other } \\
\text { bias }\end{array}$ \\
\hline $\begin{array}{l}\text { YCQ (2017) } \\
{[42]}\end{array}$ & $\mathrm{U}$ & $\mathrm{U}$ & $\mathrm{U}$ & $\mathrm{U}$ & $\mathrm{L}$ & $\mathrm{L}$ & $\mathrm{U}$ \\
\hline $\begin{array}{l}\text { LWR (2013) } \\
\text { [43] }\end{array}$ & $\mathrm{U}$ & $\mathrm{U}$ & $\mathrm{U}$ & $\mathrm{U}$ & $\mathrm{L}$ & $\mathrm{L}$ & $\mathrm{U}$ \\
\hline $\begin{array}{l}\text { WGJ (2011) } \\
{[44]}\end{array}$ & $\mathrm{U}$ & $\mathrm{U}$ & $\mathrm{U}$ & $\mathrm{U}$ & $\mathrm{L}$ & $\mathrm{L}$ & $\mathrm{U}$ \\
\hline $\begin{array}{l}\text { HRM (2013) } \\
{[45]}\end{array}$ & $\mathrm{U}$ & $\mathrm{U}$ & $\mathrm{U}$ & $\mathrm{U}$ & $\mathrm{L}$ & $\mathrm{L}$ & $\mathrm{U}$ \\
\hline $\begin{array}{l}\text { GSY (2012) } \\
{[46]}\end{array}$ & $\mathrm{U}$ & $\mathrm{U}$ & $\mathrm{U}$ & $\mathrm{U}$ & $\mathrm{L}$ & $\mathrm{L}$ & $\mathrm{U}$ \\
\hline $\begin{array}{l}\text { ZYH (2016) } \\
\text { [47] }\end{array}$ & $\mathrm{U}$ & $\mathrm{U}$ & $\mathrm{U}$ & $\mathrm{U}$ & $\mathrm{L}$ & $\mathrm{L}$ & $\mathrm{U}$ \\
\hline $\begin{array}{l}\text { ZNN (2012) } \\
{[48]}\end{array}$ & $\mathrm{U}$ & $\mathrm{U}$ & $\mathrm{U}$ & $\mathrm{U}$ & $\mathrm{L}$ & $\mathrm{L}$ & $\mathrm{U}$ \\
\hline $\begin{array}{l}\text { LJ (2014) } \\
{[49]}\end{array}$ & $\mathrm{U}$ & $\mathrm{U}$ & $\mathrm{U}$ & $\mathrm{U}$ & $\mathrm{L}$ & $\mathrm{L}$ & $\mathrm{U}$ \\
\hline \multicolumn{8}{|c|}{ Chronic pelvic inflammatory } \\
\hline $\begin{array}{l}\text { LJX2 (2014) } \\
\text { [50] }\end{array}$ & $\mathrm{U}$ & $\mathrm{U}$ & $\mathrm{U}$ & $\mathrm{U}$ & $\mathrm{L}$ & $\mathrm{L}$ & $\mathrm{U}$ \\
\hline $\begin{array}{l}\text { XZJ (2007) } \\
{[51]}\end{array}$ & $\mathrm{H}$ & $\mathrm{U}$ & $\mathrm{U}$ & $\mathrm{U}$ & $\mathrm{L}$ & $\mathrm{L}$ & $\mathrm{U}$ \\
\hline $\begin{array}{l}\text { CXC (2019) } \\
{[52]}\end{array}$ & $\mathrm{U}$ & $\mathrm{U}$ & $\mathrm{U}$ & $\mathrm{U}$ & $\mathrm{L}$ & $\mathrm{L}$ & $\mathrm{U}$ \\
\hline $\begin{array}{l}\text { DJL (2014) } \\
\text { [53] }\end{array}$ & $\mathrm{U}$ & $\mathrm{U}$ & $\mathrm{U}$ & $\mathrm{U}$ & $\mathrm{L}$ & $\mathrm{L}$ & $\mathrm{U}$ \\
\hline $\begin{array}{l}\text { PGZ (2016) } \\
{[54]}\end{array}$ & $\mathrm{H}$ & $\mathrm{U}$ & $\mathrm{U}$ & $\mathrm{U}$ & $\mathrm{L}$ & $\mathrm{L}$ & $\mathrm{U}$ \\
\hline $\begin{array}{l}\text { SXL (2015) } \\
{[55]}\end{array}$ & $\mathrm{L}$ & $\mathrm{U}$ & $\mathrm{U}$ & $\mathrm{U}$ & $\mathrm{L}$ & $\mathrm{L}$ & $\mathrm{U}$ \\
\hline $\begin{array}{l}\text { WQF (2013) } \\
\text { [56] }\end{array}$ & $\mathrm{U}$ & $\mathrm{U}$ & $\mathrm{U}$ & $\mathrm{U}$ & $\mathrm{L}$ & $\mathrm{L}$ & $\mathrm{U}$ \\
\hline $\begin{array}{l}\text { ZXJ (2015) } \\
{[57]}\end{array}$ & $\mathrm{L}$ & $\mathrm{U}$ & $\mathrm{U}$ & $\mathrm{U}$ & $\mathrm{L}$ & $\mathrm{L}$ & $\mathrm{U}$ \\
\hline $\begin{array}{l}\text { ZMY (2017) } \\
{[58]}\end{array}$ & $\mathrm{U}$ & $\mathrm{U}$ & $\mathrm{U}$ & $\mathrm{U}$ & $\mathrm{L}$ & $\mathrm{L}$ & $\mathrm{U}$ \\
\hline $\begin{array}{l}\text { LJX1 (2014) } \\
{[50]}\end{array}$ & $\mathrm{U}$ & $\mathrm{U}$ & $\mathrm{U}$ & $\mathrm{U}$ & $\mathrm{L}$ & $\mathrm{L}$ & $\mathrm{U}$ \\
\hline $\begin{array}{l}\text { LXX (2019) } \\
\text { [59] }\end{array}$ & $\mathrm{L}$ & $\mathrm{U}$ & $\mathrm{U}$ & $\mathrm{U}$ & $\mathrm{L}$ & $\mathrm{L}$ & $\mathrm{U}$ \\
\hline $\begin{array}{l}\text { LH (2019) } \\
{[60]}\end{array}$ & $\mathrm{U}$ & $\mathrm{U}$ & $\mathrm{U}$ & $\mathrm{U}$ & $\mathrm{L}$ & $\mathrm{L}$ & $\mathrm{U}$ \\
\hline $\begin{array}{l}\text { PJQ (2019) } \\
\text { [61] }\end{array}$ & $\mathrm{U}$ & $\mathrm{U}$ & $\mathrm{U}$ & $\mathrm{U}$ & $\mathrm{L}$ & $\mathrm{L}$ & $\mathrm{U}$ \\
\hline $\begin{array}{l}\text { SWA (2014) } \\
\text { [62] }\end{array}$ & $\mathrm{U}$ & $\mathrm{U}$ & $\mathrm{U}$ & $\mathrm{U}$ & $\mathrm{L}$ & $\mathrm{L}$ & $\mathrm{U}$ \\
\hline $\begin{array}{l}\text { SQB2 (2012) } \\
\text { [63] }\end{array}$ & $\mathrm{U}$ & $\mathrm{U}$ & $\mathrm{U}$ & $\mathrm{U}$ & $\mathrm{L}$ & $\mathrm{L}$ & $\mathrm{U}$ \\
\hline $\begin{array}{l}\mathrm{ZY}(2014) \\
{[64]}\end{array}$ & $\mathrm{U}$ & $\mathrm{U}$ & $\mathrm{U}$ & $\mathrm{U}$ & $\mathrm{L}$ & $\mathrm{L}$ & $\mathrm{U}$ \\
\hline $\begin{array}{l}\text { SQB1 (2012) } \\
\text { [63] }\end{array}$ & $\mathrm{U}$ & $\mathrm{U}$ & $\mathrm{U}$ & $\mathrm{U}$ & $\mathrm{L}$ & $\mathrm{L}$ & $\mathrm{U}$ \\
\hline $\begin{array}{l}\text { SPF (2015) } \\
{[65]}\end{array}$ & $\mathrm{U}$ & $\mathrm{U}$ & $\mathrm{U}$ & $\mathrm{U}$ & $\mathrm{L}$ & $\mathrm{L}$ & $\mathrm{U}$ \\
\hline $\begin{array}{l}\text { CF (2018) } \\
{[66]}\end{array}$ & $\mathrm{U}$ & $\mathrm{U}$ & $\mathrm{U}$ & $\mathrm{U}$ & $\mathrm{L}$ & $\mathrm{L}$ & $\mathrm{U}$ \\
\hline $\begin{array}{l}\text { DLL (2017) } \\
\text { [67] }\end{array}$ & $\mathrm{U}$ & $\mathrm{U}$ & $\mathrm{U}$ & $\mathrm{U}$ & $\mathrm{L}$ & $\mathrm{L}$ & $\mathrm{U}$ \\
\hline
\end{tabular}


TABLE 3: Continued.

\begin{tabular}{|c|c|c|c|c|c|c|c|}
\hline Study ID & $\begin{array}{c}\text { Random } \\
\text { sequence } \\
\text { generation } \\
\text { (selection bias) }\end{array}$ & $\begin{array}{c}\text { Allocation } \\
\text { concealment } \\
\text { (selection bias) }\end{array}$ & $\begin{array}{c}\text { Blinding of } \\
\text { participants and } \\
\text { personnel } \\
\text { (performance bias) }\end{array}$ & $\begin{array}{c}\text { Blinding of } \\
\text { outcome } \\
\text { assessment } \\
\text { (detection bias) }\end{array}$ & $\begin{array}{c}\text { Incomplete } \\
\text { outcome data } \\
\text { (attrition bias) }\end{array}$ & $\begin{array}{l}\text { Selective } \\
\text { reporting } \\
\text { (reporting } \\
\text { bias) }\end{array}$ & $\begin{array}{l}\text { Other } \\
\text { bias }\end{array}$ \\
\hline $\begin{array}{l}\text { FJY (2010) } \\
\text { [68] }\end{array}$ & $\mathrm{U}$ & $\mathrm{U}$ & U & $\mathrm{U}$ & $\mathrm{L}$ & $\mathrm{L}$ & $\mathrm{U}$ \\
\hline $\begin{array}{l}\text { FP (2013) } \\
\text { [69] }\end{array}$ & $\mathrm{U}$ & $\mathrm{U}$ & $\mathrm{U}$ & U & $\mathrm{L}$ & $\mathrm{L}$ & $\mathrm{U}$ \\
\hline $\begin{array}{l}\text { KY (2018) } \\
{[70]}\end{array}$ & U & U & U & U & $\mathrm{L}$ & $\mathrm{L}$ & $\mathrm{U}$ \\
\hline $\begin{array}{l}\text { LMY (2016) } \\
{[71]}\end{array}$ & U & U & $\mathrm{U}$ & U & $\mathrm{L}$ & $\mathrm{L}$ & $\mathrm{U}$ \\
\hline $\begin{array}{l}\text { LYK (2016) } \\
\text { [72] }\end{array}$ & U & U & $\mathrm{U}$ & $\mathrm{U}$ & $\mathrm{L}$ & $\mathrm{L}$ & $\mathrm{U}$ \\
\hline $\begin{array}{l}\text { LongH } \\
\text { (2011) [73] }\end{array}$ & $\mathrm{U}$ & $\mathrm{U}$ & $\mathrm{U}$ & $\mathrm{U}$ & $\mathrm{L}$ & $\mathrm{L}$ & $\mathrm{U}$ \\
\hline $\begin{array}{l}\text { RDQ (2019) } \\
{[74]}\end{array}$ & $\mathrm{U}$ & $\mathrm{U}$ & $\mathrm{U}$ & $\mathrm{U}$ & $\mathrm{L}$ & $\mathrm{L}$ & $\mathrm{U}$ \\
\hline $\begin{array}{l}\text { ShaoXL } \\
\text { (2012) [75] }\end{array}$ & U & U & U & U & $\mathrm{L}$ & $\mathrm{L}$ & $\mathrm{U}$ \\
\hline $\begin{array}{l}\text { TXG (2015) } \\
{[76]}\end{array}$ & U & $\mathrm{U}$ & $\mathrm{U}$ & U & $\mathrm{L}$ & $\mathrm{L}$ & $\mathrm{U}$ \\
\hline $\begin{array}{l}\text { WJP (2013) } \\
\text { [77] }\end{array}$ & $\mathrm{U}$ & $\mathrm{U}$ & $\mathrm{U}$ & $\mathrm{U}$ & $\mathrm{H}$ & $\mathrm{L}$ & $\mathrm{U}$ \\
\hline $\begin{array}{l}\text { YM (2017) } \\
\text { [78] }\end{array}$ & $\mathrm{U}$ & $\mathrm{U}$ & $\mathrm{U}$ & $\mathrm{U}$ & $\mathrm{L}$ & $\mathrm{L}$ & $\mathrm{U}$ \\
\hline $\begin{array}{l}\text { YQ (2014) } \\
\text { [79] }\end{array}$ & $\mathrm{U}$ & U & $\mathrm{U}$ & $\mathrm{U}$ & $\mathrm{L}$ & $\mathrm{L}$ & $\mathrm{U}$ \\
\hline $\begin{array}{l}\text { ZYL (2017) } \\
\text { [80] }\end{array}$ & $\mathrm{L}$ & U & U & U & $\mathrm{L}$ & $\mathrm{L}$ & U \\
\hline $\begin{array}{l}\text { DLH (2019) } \\
\text { [81] }\end{array}$ & $\mathrm{H}$ & $\mathrm{U}$ & $\mathrm{U}$ & $\mathrm{U}$ & $\mathrm{L}$ & $\mathrm{L}$ & $\mathrm{U}$ \\
\hline $\begin{array}{l}\text { HJY (2019) } \\
\text { [82] }\end{array}$ & $\mathrm{L}$ & U & $\mathrm{U}$ & $\mathrm{U}$ & $\mathrm{L}$ & $\mathrm{L}$ & $\mathrm{U}$ \\
\hline $\begin{array}{l}\text { LJ (2019) } \\
{[83]}\end{array}$ & $\mathrm{U}$ & $\mathrm{L}$ & $\mathrm{L}$ & $\mathrm{U}$ & $\mathrm{L}$ & $\mathrm{L}$ & $\mathrm{U}$ \\
\hline $\begin{array}{l}\text { SJL (2016) } \\
{[84]}\end{array}$ & $\mathrm{U}$ & U & $\mathrm{U}$ & $\mathrm{U}$ & $\mathrm{L}$ & $\mathrm{L}$ & $\mathrm{U}$ \\
\hline $\begin{array}{l}\text { WJ (2018) } \\
{[85]}\end{array}$ & $\mathrm{L}$ & $\mathrm{U}$ & $\mathrm{U}$ & $\mathrm{U}$ & $\mathrm{L}$ & $\mathrm{L}$ & $\mathrm{U}$ \\
\hline $\begin{array}{l}\text { ZL (2015) } \\
{[86]}\end{array}$ & $\mathrm{U}$ & $\mathrm{U}$ & $\mathrm{U}$ & U & $\mathrm{L}$ & $\mathrm{L}$ & $\mathrm{U}$ \\
\hline $\begin{array}{l}\text { ZT (2018) } \\
\text { [87] }\end{array}$ & $\mathrm{U}$ & $\mathrm{U}$ & $\mathrm{U}$ & U & $\mathrm{L}$ & $\mathrm{L}$ & $\mathrm{U}$ \\
\hline $\begin{array}{l}\text { ZhaoJ } \\
\text { (2017) [88] }\end{array}$ & U & U & $\mathrm{U}$ & $\mathrm{U}$ & $\mathrm{L}$ & $\mathrm{L}$ & $\mathrm{U}$ \\
\hline $\begin{array}{l}\text { ZengJ } \\
\text { (2016) [89] }\end{array}$ & $\mathrm{U}$ & $\mathrm{U}$ & $\mathrm{U}$ & $\mathrm{U}$ & $\mathrm{L}$ & $\mathrm{L}$ & $\mathrm{U}$ \\
\hline $\begin{array}{l}\text { CXF (2018) } \\
\text { [90] }\end{array}$ & $\mathrm{U}$ & U & $\mathrm{U}$ & U & $\mathrm{L}$ & $\mathrm{L}$ & $\mathrm{U}$ \\
\hline $\begin{array}{l}\text { DCM (2016) } \\
\text { [91] }\end{array}$ & $\mathrm{U}$ & $\mathrm{U}$ & $\mathrm{U}$ & $\mathrm{U}$ & $\mathrm{L}$ & $\mathrm{L}$ & $\mathrm{U}$ \\
\hline $\begin{array}{l}\text { DQY (2015) } \\
\text { [92] }\end{array}$ & $\mathrm{U}$ & $\mathrm{U}$ & $\mathrm{U}$ & $\mathrm{U}$ & $\mathrm{L}$ & $\mathrm{L}$ & $\mathrm{U}$ \\
\hline $\begin{array}{l}\text { HSX (2019) } \\
\text { [93] }\end{array}$ & $\mathrm{H}$ & $\mathrm{U}$ & $\mathrm{U}$ & U & $\mathrm{L}$ & $\mathrm{L}$ & $\mathrm{U}$ \\
\hline $\begin{array}{l}\text { HHJ (2019) } \\
\text { [94] }\end{array}$ & $\mathrm{L}$ & U & $\mathrm{U}$ & U & $\mathrm{L}$ & $\mathrm{L}$ & U \\
\hline $\begin{array}{l}\text { HHM } \\
\text { (2019) [95] }\end{array}$ & $\mathrm{L}$ & U & $\mathrm{U}$ & $\mathrm{U}$ & $\mathrm{L}$ & $\mathrm{L}$ & $\mathrm{U}$ \\
\hline
\end{tabular}


TABle 3: Continued.

\begin{tabular}{|c|c|c|c|c|c|c|c|}
\hline Study ID & $\begin{array}{c}\text { Random } \\
\text { sequence } \\
\text { generation } \\
\text { (selection bias) }\end{array}$ & $\begin{array}{c}\text { Allocation } \\
\text { concealment } \\
\text { (selection bias) }\end{array}$ & $\begin{array}{c}\text { Blinding of } \\
\text { participants and } \\
\text { personnel } \\
\text { (performance bias) }\end{array}$ & $\begin{array}{c}\text { Blinding of } \\
\text { outcome } \\
\text { assessment } \\
\text { (detection bias) }\end{array}$ & $\begin{array}{c}\text { Incomplete } \\
\text { outcome data } \\
\text { (attrition bias) }\end{array}$ & $\begin{array}{l}\text { Selective } \\
\text { reporting } \\
\text { (reporting } \\
\text { bias) }\end{array}$ & $\begin{array}{c}\text { Other } \\
\text { bias }\end{array}$ \\
\hline $\begin{array}{l}\text { HYL (2015) } \\
{[96]}\end{array}$ & $\mathrm{L}$ & $\mathrm{U}$ & $\mathrm{U}$ & $\mathrm{U}$ & $\mathrm{L}$ & $\mathrm{L}$ & $\mathrm{U}$ \\
\hline $\begin{array}{l}\text { LML (2019) } \\
\text { [97] }\end{array}$ & $\mathrm{U}$ & $\mathrm{U}$ & $\mathrm{U}$ & $\mathrm{U}$ & $\mathrm{L}$ & $\mathrm{L}$ & $\mathrm{U}$ \\
\hline $\begin{array}{l}\text { LHR (2013) } \\
\text { [98] }\end{array}$ & $\mathrm{U}$ & $\mathrm{U}$ & $\mathrm{U}$ & $\mathrm{U}$ & $\mathrm{L}$ & $\mathrm{L}$ & $\mathrm{U}$ \\
\hline $\begin{array}{l}\text { LXL (2018) } \\
\text { [99] }\end{array}$ & $\mathrm{L}$ & $\mathrm{U}$ & $\mathrm{U}$ & $\mathrm{U}$ & $\mathrm{L}$ & $\mathrm{L}$ & $\mathrm{U}$ \\
\hline $\begin{array}{l}\text { LSY (2018) } \\
{[100]}\end{array}$ & $\mathrm{L}$ & $\mathrm{U}$ & $\mathrm{U}$ & $\mathrm{U}$ & $\mathrm{L}$ & $\mathrm{L}$ & $\mathrm{U}$ \\
\hline $\begin{array}{l}\text { LYZ (2012) } \\
\text { [101] }\end{array}$ & $\mathrm{H}$ & $\mathrm{U}$ & $\mathrm{U}$ & $\mathrm{U}$ & $\mathrm{L}$ & $\mathrm{L}$ & $\mathrm{U}$ \\
\hline $\begin{array}{l}\text { MYQ (2019) } \\
{[102]}\end{array}$ & $\mathrm{U}$ & $\mathrm{U}$ & $\mathrm{U}$ & $\mathrm{U}$ & $\mathrm{L}$ & $\mathrm{L}$ & $\mathrm{U}$ \\
\hline $\begin{array}{l}\mathrm{RC}(2016) \\
{[103]}\end{array}$ & $\mathrm{U}$ & $\mathrm{U}$ & $\mathrm{U}$ & $\mathrm{U}$ & $\mathrm{L}$ & $\mathrm{L}$ & $\mathrm{U}$ \\
\hline $\begin{array}{l}\text { RL (2017) } \\
{[104]}\end{array}$ & $\mathrm{L}$ & $\mathrm{U}$ & $\mathrm{U}$ & $\mathrm{U}$ & $\mathrm{L}$ & $\mathrm{L}$ & $\mathrm{U}$ \\
\hline $\begin{array}{l}\text { SNY.HSY } \\
(2016)[105]\end{array}$ & $\mathrm{U}$ & $\mathrm{U}$ & $\mathrm{U}$ & $\mathrm{U}$ & $\mathrm{L}$ & $\mathrm{L}$ & $\mathrm{U}$ \\
\hline $\begin{array}{l}\text { SQJ (2012) } \\
{[106]}\end{array}$ & $\mathrm{U}$ & $\mathrm{U}$ & $\mathrm{U}$ & $\mathrm{U}$ & $\mathrm{L}$ & $\mathrm{L}$ & $\mathrm{U}$ \\
\hline $\begin{array}{l}\text { YLH (2012) } \\
{[107]}\end{array}$ & $\mathrm{U}$ & $\mathrm{U}$ & $\mathrm{U}$ & $\mathrm{U}$ & $\mathrm{L}$ & $\mathrm{L}$ & $\mathrm{U}$ \\
\hline $\begin{array}{l}\text { WDD } \\
(2017)[108]\end{array}$ & $\mathrm{L}$ & $\mathrm{U}$ & $\mathrm{U}$ & $\mathrm{U}$ & $\mathrm{L}$ & $\mathrm{L}$ & $\mathrm{U}$ \\
\hline $\begin{array}{l}\text { WHL (2011) } \\
\text { [109] }\end{array}$ & $\mathrm{U}$ & $\mathrm{U}$ & $\mathrm{U}$ & $\mathrm{U}$ & $\mathrm{L}$ & $\mathrm{L}$ & $\mathrm{U}$ \\
\hline $\begin{array}{l}\text { WLY (2019) } \\
\text { [110] }\end{array}$ & $\mathrm{U}$ & $\mathrm{U}$ & $\mathrm{U}$ & $\mathrm{U}$ & $\mathrm{L}$ & $\mathrm{L}$ & $\mathrm{U}$ \\
\hline $\begin{array}{l}\text { WSY (2011) } \\
{[111]}\end{array}$ & $\mathrm{U}$ & $\mathrm{U}$ & $\mathrm{U}$ & $\mathrm{U}$ & $\mathrm{L}$ & $\mathrm{L}$ & $\mathrm{U}$ \\
\hline $\begin{array}{l}\text { WY (2018) } \\
{[112]}\end{array}$ & $\mathrm{L}$ & $\mathrm{U}$ & $\mathrm{U}$ & $\mathrm{U}$ & $\mathrm{L}$ & $\mathrm{L}$ & $\mathrm{U}$ \\
\hline $\begin{array}{l}\text { WDM } \\
\text { (2019) [113] }\end{array}$ & $\mathrm{U}$ & $\mathrm{U}$ & $\mathrm{U}$ & $\mathrm{U}$ & $\mathrm{L}$ & $\mathrm{L}$ & $\mathrm{U}$ \\
\hline $\begin{array}{l}\text { XW (2015) } \\
\text { [114] }\end{array}$ & $\mathrm{U}$ & $\mathrm{U}$ & $\mathrm{U}$ & $\mathrm{U}$ & $\mathrm{L}$ & $\mathrm{L}$ & $\mathrm{U}$ \\
\hline $\begin{array}{l}\text { XRQ (2018) } \\
\text { [115] }\end{array}$ & $\mathrm{U}$ & $\mathrm{U}$ & $\mathrm{U}$ & $\mathrm{U}$ & $\mathrm{L}$ & $\mathrm{L}$ & $\mathrm{U}$ \\
\hline $\begin{array}{l}\text { YDM (2015) } \\
{[116]}\end{array}$ & $\mathrm{U}$ & $\mathrm{U}$ & $\mathrm{U}$ & $\mathrm{U}$ & $\mathrm{L}$ & $\mathrm{L}$ & $\mathrm{U}$ \\
\hline $\begin{array}{l}\text { YXH (2016) } \\
\text { [117] }\end{array}$ & $\mathrm{L}$ & $\mathrm{U}$ & $\mathrm{U}$ & $\mathrm{U}$ & $\mathrm{L}$ & $\mathrm{L}$ & $\mathrm{U}$ \\
\hline $\begin{array}{l}\text { ZhangJ } \\
(2017) \text { [118] }\end{array}$ & $\mathrm{U}$ & $\mathrm{U}$ & $\mathrm{U}$ & $\mathrm{U}$ & $\mathrm{L}$ & $\mathrm{L}$ & $\mathrm{U}$ \\
\hline \multicolumn{8}{|l|}{ Endometritis } \\
\hline $\begin{array}{l}\text { SFF (2017) } \\
{[119]}\end{array}$ & $\mathrm{U}$ & $\mathrm{U}$ & $\mathrm{U}$ & $\mathrm{U}$ & $\mathrm{L}$ & $\mathrm{L}$ & $\mathrm{U}$ \\
\hline $\begin{array}{l}\text { SCP (2019) } \\
{[120]}\end{array}$ & $\mathrm{U}$ & $\mathrm{U}$ & $\mathrm{U}$ & $\mathrm{U}$ & $\mathrm{L}$ & $\mathrm{L}$ & $\mathrm{U}$ \\
\hline $\begin{array}{l}\text { DYY (2019) } \\
\text { [121] }\end{array}$ & $\mathrm{U}$ & $\mathrm{U}$ & $\mathrm{U}$ & $\mathrm{U}$ & $\mathrm{L}$ & $\mathrm{L}$ & $\mathrm{U}$ \\
\hline $\begin{array}{l}\text { LY (2019) } \\
{[122]}\end{array}$ & $\mathrm{U}$ & $\mathrm{U}$ & $\mathrm{U}$ & $\mathrm{U}$ & $\mathrm{L}$ & $\mathrm{L}$ & $\mathrm{U}$ \\
\hline $\begin{array}{l}\text { HHF (2017) } \\
{[123]}\end{array}$ & $\mathrm{L}$ & $\mathrm{U}$ & $\mathrm{U}$ & $\mathrm{U}$ & $\mathrm{L}$ & $\mathrm{L}$ & $\mathrm{U}$ \\
\hline
\end{tabular}


TABle 3: Continued.

\begin{tabular}{|c|c|c|c|c|c|c|c|}
\hline Study ID & $\begin{array}{c}\text { Random } \\
\text { sequence } \\
\text { generation } \\
\text { (selection bias) }\end{array}$ & $\begin{array}{c}\text { Allocation } \\
\text { concealment } \\
\text { (selection bias) }\end{array}$ & $\begin{array}{c}\text { Blinding of } \\
\text { participants and } \\
\text { personnel } \\
\text { (performance bias) }\end{array}$ & $\begin{array}{c}\text { Blinding of } \\
\text { outcome } \\
\text { assessment } \\
\text { (detection bias) }\end{array}$ & $\begin{array}{c}\text { Incomplete } \\
\text { outcome data } \\
\text { (attrition bias) }\end{array}$ & $\begin{array}{l}\text { Selective } \\
\text { reporting } \\
\text { (reporting } \\
\text { bias) }\end{array}$ & $\begin{array}{c}\text { Other } \\
\text { bias }\end{array}$ \\
\hline $\begin{array}{l}\text { WJF (2019) } \\
\text { [124] }\end{array}$ & $\mathrm{U}$ & $\mathrm{U}$ & $\mathrm{U}$ & U & $\mathrm{L}$ & $\mathrm{L}$ & $\mathrm{U}$ \\
\hline $\begin{array}{l}\text { MTX (2016) } \\
{[125]}\end{array}$ & $\mathrm{U}$ & $\mathrm{U}$ & $\mathrm{U}$ & $\mathrm{U}$ & $\mathrm{L}$ & $\mathrm{L}$ & $\mathrm{U}$ \\
\hline $\begin{array}{l}\text { SXW (2018) } \\
{[126]}\end{array}$ & $\mathrm{L}$ & $\mathrm{U}$ & U & U & $\mathrm{L}$ & $\mathrm{L}$ & $\mathrm{U}$ \\
\hline $\begin{array}{l}\text { ZHH (2018) } \\
\text { [127] }\end{array}$ & $\mathrm{L}$ & $\mathrm{U}$ & $\mathrm{U}$ & U & $\mathrm{L}$ & $\mathrm{L}$ & $\mathrm{U}$ \\
\hline $\begin{array}{l}\text { ZL (2019) } \\
{[128]}\end{array}$ & $\mathrm{L}$ & $\mathrm{U}$ & U & $\mathrm{U}$ & $\mathrm{L}$ & $\mathrm{L}$ & $\mathrm{U}$ \\
\hline $\begin{array}{l}\text { DCL (2017) } \\
\text { [129] }\end{array}$ & $\mathrm{U}$ & $\mathrm{U}$ & $\mathrm{U}$ & U & $\mathrm{L}$ & $\mathrm{L}$ & $\mathrm{U}$ \\
\hline $\begin{array}{l}\text { DFY (2017) } \\
{[130]}\end{array}$ & $\mathrm{L}$ & $\mathrm{U}$ & $\mathrm{U}$ & $\mathrm{U}$ & $\mathrm{L}$ & $\mathrm{L}$ & $\mathrm{U}$ \\
\hline $\begin{array}{l}\text { GY(2015) } \\
{[131]}\end{array}$ & $\mathrm{L}$ & $\mathrm{U}$ & U & U & $\mathrm{L}$ & $\mathrm{L}$ & $\mathrm{U}$ \\
\hline $\begin{array}{l}\text { LWP (2019) } \\
\text { [132] }\end{array}$ & $\mathrm{L}$ & $\mathrm{U}$ & $\mathrm{U}$ & $\mathrm{U}$ & $\mathrm{L}$ & $\mathrm{L}$ & $\mathrm{U}$ \\
\hline $\begin{array}{l}\text { MYJ (2018) } \\
\text { [133] }\end{array}$ & $\mathrm{L}$ & $\mathrm{U}$ & $\mathrm{U}$ & $\mathrm{U}$ & $\mathrm{L}$ & $\mathrm{L}$ & $\mathrm{U}$ \\
\hline $\begin{array}{l}\text { DFL (2017) } \\
{[134]}\end{array}$ & $\mathrm{U}$ & $\mathrm{U}$ & $\mathrm{U}$ & $\mathrm{U}$ & $\mathrm{L}$ & $\mathrm{L}$ & $\mathrm{U}$ \\
\hline $\begin{array}{l}\text { HXY (2018) } \\
\text { [135] }\end{array}$ & $\mathrm{U}$ & $\mathrm{U}$ & $\mathrm{U}$ & $\mathrm{U}$ & $\mathrm{L}$ & $\mathrm{L}$ & $\mathrm{U}$ \\
\hline $\begin{array}{l}\text { ZQ (2019) } \\
{[136]}\end{array}$ & $\mathrm{U}$ & $\mathrm{U}$ & $\mathrm{U}$ & $\mathrm{U}$ & $\mathrm{L}$ & $\mathrm{L}$ & $\mathrm{U}$ \\
\hline $\begin{array}{l}\text { LXZ (2017) } \\
\text { [137] }\end{array}$ & $\mathrm{U}$ & $\mathrm{U}$ & $\mathrm{U}$ & $\mathrm{U}$ & $\mathrm{L}$ & $\mathrm{L}$ & $\mathrm{U}$ \\
\hline $\begin{array}{l}\text { CXH (2017) } \\
{[138]}\end{array}$ & $\mathrm{L}$ & $\mathrm{U}$ & $\mathrm{U}$ & $\mathrm{U}$ & $\mathrm{L}$ & $\mathrm{L}$ & $\mathrm{U}$ \\
\hline $\begin{array}{l}\text { FCF (2016) } \\
{[139]}\end{array}$ & $\mathrm{U}$ & $\mathrm{U}$ & $\mathrm{U}$ & $\mathrm{U}$ & $\mathrm{L}$ & $\mathrm{L}$ & $\mathrm{U}$ \\
\hline $\begin{array}{l}\text { LBY (2019) } \\
\text { [140] }\end{array}$ & $\mathrm{U}$ & $\mathrm{U}$ & $\mathrm{U}$ & $\mathrm{U}$ & $\mathrm{L}$ & $\mathrm{L}$ & $\mathrm{U}$ \\
\hline $\begin{array}{l}\text { LSQ (2017) } \\
\text { [141] }\end{array}$ & $\mathrm{L}$ & $\mathrm{U}$ & $\mathrm{U}$ & U & $\mathrm{L}$ & $\mathrm{L}$ & $\mathrm{U}$ \\
\hline $\begin{array}{l}\text { YL (2018) } \\
{[142]}\end{array}$ & $\mathrm{U}$ & $\mathrm{U}$ & $\mathrm{U}$ & $\mathrm{U}$ & $\mathrm{L}$ & $\mathrm{L}$ & $\mathrm{U}$ \\
\hline $\begin{array}{l}\text { ZQ (2016) } \\
{[143]}\end{array}$ & $\mathrm{U}$ & $\mathrm{U}$ & $\mathrm{U}$ & $\mathrm{U}$ & $\mathrm{L}$ & $\mathrm{L}$ & $\mathrm{U}$ \\
\hline $\begin{array}{l}\text { ZCY (2018) } \\
{[144]}\end{array}$ & $\mathrm{U}$ & $\mathrm{U}$ & $\mathrm{U}$ & $\mathrm{U}$ & $\mathrm{L}$ & $\mathrm{L}$ & $\mathrm{U}$ \\
\hline $\begin{array}{l}\text { ZGB (2017) } \\
\text { [145] }\end{array}$ & $\mathrm{U}$ & $\mathrm{U}$ & $\mathrm{U}$ & $\mathrm{U}$ & $\mathrm{L}$ & $\mathrm{L}$ & $\mathrm{U}$ \\
\hline $\begin{array}{l}\text { GLJNT } \\
\text { (2017) [146] }\end{array}$ & $\mathrm{U}$ & $\mathrm{U}$ & $\mathrm{U}$ & $\mathrm{U}$ & $\mathrm{L}$ & $\mathrm{L}$ & $\mathrm{U}$ \\
\hline $\begin{array}{l}\text { CJH (2016) } \\
{[147]}\end{array}$ & $\mathrm{U}$ & $\mathrm{U}$ & $\mathrm{U}$ & $\mathrm{U}$ & $\mathrm{L}$ & $\mathrm{L}$ & $\mathrm{U}$ \\
\hline $\begin{array}{l}\text { AYGL } \\
\text { (2018) [148] }\end{array}$ & $\mathrm{L}$ & $\mathrm{U}$ & $\mathrm{U}$ & $\mathrm{U}$ & $\mathrm{L}$ & $\mathrm{L}$ & $\mathrm{U}$ \\
\hline $\begin{array}{l}\text { SXX (2018) } \\
{[149]}\end{array}$ & $\mathrm{U}$ & $\mathrm{U}$ & $\mathrm{U}$ & U & $\mathrm{L}$ & $\mathrm{L}$ & $\mathrm{U}$ \\
\hline $\begin{array}{l}\text { XCY (2015) } \\
\text { [150] }\end{array}$ & $\mathrm{L}$ & U & $\mathrm{U}$ & $\mathrm{U}$ & $\mathrm{L}$ & $\mathrm{L}$ & $\mathrm{U}$ \\
\hline $\begin{array}{l}\text { HF (2016) } \\
{[151]}\end{array}$ & $\mathrm{L}$ & $\mathrm{U}$ & $\mathrm{U}$ & $\mathrm{U}$ & $\mathrm{L}$ & $\mathrm{L}$ & $\mathrm{U}$ \\
\hline
\end{tabular}

L, low risk; $U$, unclear risk; $H$, high risk. 


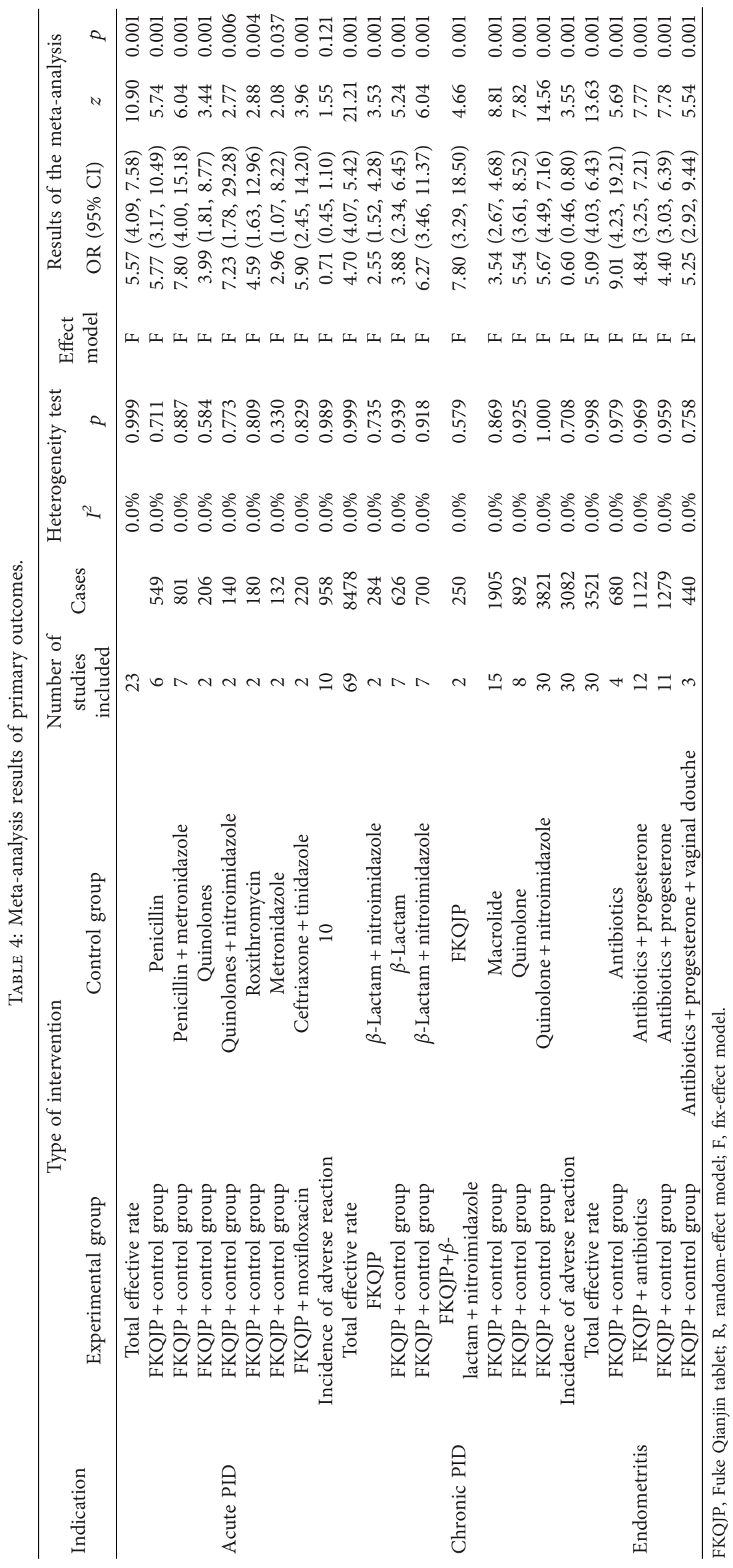




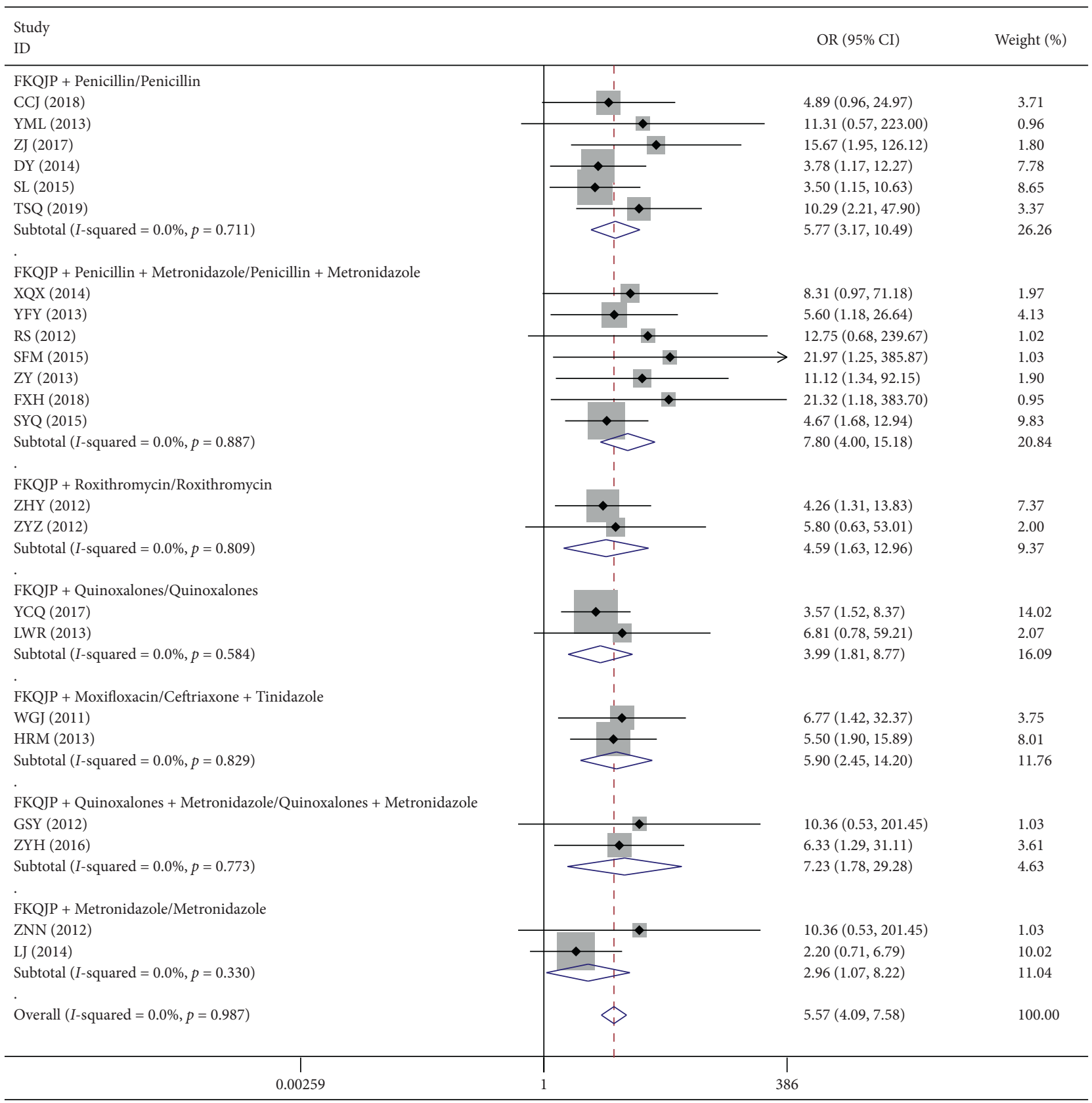

Figure 2: Meta-analysis of total effective rate of acute pelvic inflammatory disease.

outcome. The results of the fixed-effect model showed that the combined group of Fuke Qianjin tablets had a better total effective rate of treating endometritis than the control group $(\mathrm{OR}=5.09, \quad 95 \% \quad \mathrm{CI} 4.03-6.43, \quad Z=13.63, \quad p=0.001)$. According to the specific intervention measures, the subgroup analysis showed that the combined group of Fuke Qianjin tablets had a better curative effect than the control group (Table 4 and Figure 8 ).

(2) Adverse Reaction. In the included study, only 4 studies (a total of 427 participants) counted adverse reactions as part of the study. 3 studies reported no serious adverse reactions or no obvious adverse reactions, and 1 reported specific adverse reactions: there were 2 cases in the trial group ( 1 case of nausea and 1 case of gastrointestinal reaction); there were 3 cases in the control group (1 case of nausea, 1 case of vomiting, and 1 case of gastrointestinal reaction). There was no significant difference in the incidence of adverse reactions between the two groups $(p>0.05)$.

3.4.2. Secondary Outcomes. Comparisons in secondary outcomes were also made. Similar to the primary outcome, Fuke Qianjin tablets combined with antibiotics had better efficacy profiles in improving endometrial thickness, menstrual recovery, and relapse rate, as shown in Table 5. 


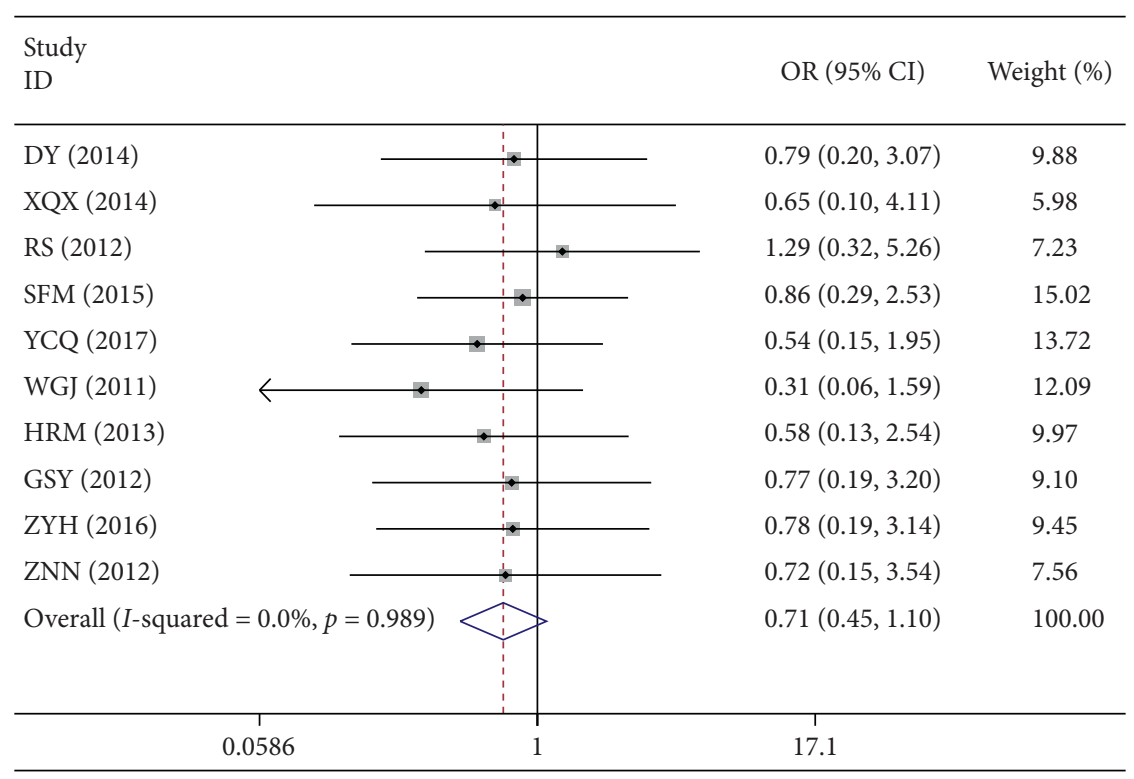

FIGURE 3: Meta-analysis of adverse reaction of acute pelvic inflammatory disease.

TABle 5: The meta-analysis results of the secondary outcomes.

\begin{tabular}{|c|c|c|c|c|c|c|c|c|}
\hline \multirow{2}{*}{ Indication } & \multirow{2}{*}{ Outcomes } & \multirow{2}{*}{$\begin{array}{l}\text { Number of } \\
\text { studies } \\
\text { included }\end{array}$} & \multirow{2}{*}{ Cases } & \multicolumn{2}{|c|}{$\begin{array}{l}\text { Heterogeneity } \\
\text { test }\end{array}$} & \multirow{2}{*}{$\begin{array}{l}\text { Effect } \\
\text { model }\end{array}$} & \multicolumn{2}{|c|}{ Results of the meta-analysis } \\
\hline & & & & $I^{2}$ & $p$ & & $\begin{array}{l}\text { SMD/OR } \\
(95 \% \mathrm{CI})\end{array}$ & $p$ \\
\hline \multirow{8}{*}{ Acute PID } & $\begin{array}{c}\text { Clinical symptom remission or } \\
\text { disappearance time }\end{array}$ & 8 & 899 & - & - & - & - & - \\
\hline & The time of the masses disappearance & 7 & 821 & $89.2 \%$ & 0.001 & $\mathrm{R}$ & $-1.36(-1.84,-0.89)$ & 0.001 \\
\hline & $\begin{array}{c}\text { The time of body temperature return } \\
\text { to normal }\end{array}$ & 7 & 821 & $84.9 \%$ & 0.001 & $\mathrm{R}$ & $-1.19(-1.58,-0.79)$ & 0.001 \\
\hline & The time to relieve abdominal pain & 7 & 821 & $90.7 \%$ & 0.001 & $\mathrm{R}$ & $-1.83(-2.38,-1.29)$ & 0.001 \\
\hline & $\begin{array}{l}\text { Serum inflammatory factor } \\
\text { concentration }\end{array}$ & 6 & 558 & - & - & - & - & - \\
\hline & CRP concentration & 3 & 300 & $92.1 \%$ & 0.001 & $\mathrm{R}$ & $-1.99(-3.01,-0.98)$ & 0.001 \\
\hline & TNF- $\alpha$ concentration & 4 & 372 & $50.8 \%$ & 0.107 & $\mathrm{~F}$ & $-1.73(-1.97,-1.49)$ & 0.001 \\
\hline & IL-6 concentration & 4 & 380 & $94.4 \%$ & 0.001 & $\mathrm{R}$ & $-1.53(-2.52,-0.53)$ & 0.001 \\
\hline \multirow{10}{*}{ Chronic PID } & $\begin{array}{l}\text { Clinical symptom remission or } \\
\text { disappearance time }\end{array}$ & 13 & 1394 & - & - & - & - & - \\
\hline & The remission time of uterine pain & 5 & 542 & $92.0 \%$ & 0.001 & $\mathrm{R}$ & $-2.48(-3.29,-1.66)$ & 0.001 \\
\hline & $\begin{array}{l}\text { The remission time of abdominal } \\
\text { pain }\end{array}$ & 12 & 1208 & $85.6 \%$ & 0.001 & $\mathrm{R}$ & $-2.12(-2.49,-1.74)$ & 0.001 \\
\hline & $\begin{array}{c}\text { The time for leucorrhoea to return to } \\
\text { normal }\end{array}$ & 7 & 782 & $27.4 \%$ & 0.219 & $\mathrm{~F}$ & $-1.38(-1.54,-1.22)$ & 0.001 \\
\hline & $\begin{array}{l}\text { Serum inflammatory factor } \\
\text { concentration }\end{array}$ & 11 & 2317 & - & - & - & - & - \\
\hline & CRP concentration & 5 & 1575 & $87.6 \%$ & 0.001 & $\mathrm{R}$ & $-3.10(-3.69,-2.51)$ & 0.001 \\
\hline & TNF- $\alpha$ concentration & 5 & 646 & $96.6 \%$ & 0.001 & $\mathrm{R}$ & $-2.56(-3.68,-1.43)$ & 0.001 \\
\hline & IL-2 concentration & 3 & 264 & $0.0 \%$ & 0.969 & $\mathrm{~F}$ & $0.85(0.59,1.10)$ & 0.001 \\
\hline & IL-4 concentration & 3 & 264 & $0.0 \%$ & 0.933 & $\mathrm{~F}$ & $0.60(0.59,0.85)$ & 0.001 \\
\hline & IL-10 concentration & 2 & 182 & $0.0 \%$ & 0.790 & $\mathrm{~F}$ & $0.49(0.20,0.79)$ & 0.001 \\
\hline \multirow{6}{*}{ Endometritis } & Endometrial thickness & 16 & 1680 & $87.8 \%$ & 0.001 & $\mathrm{R}$ & $2.25(1.89,2.60)$ & 0.001 \\
\hline & Normalization rate of menstrual & 12 & 1413 & $0.0 \%$ & 0.978 & $\mathrm{~F}$ & $4.01(3.04,5.30)$ & 0.001 \\
\hline & $\begin{array}{l}\text { Normalization rate of menstrual } \\
\text { cycle }\end{array}$ & 6 & 845 & $46.3 \%$ & 0.097 & $\mathrm{~F}$ & $5.57(3.82,8.13)$ & 0.001 \\
\hline & $\begin{array}{c}\text { Normalization rate of menstrual } \\
\text { amount }\end{array}$ & 6 & 865 & $79.9 \%$ & 0.001 & $\mathrm{R}$ & $4.96(1.72,14.28)$ & 0.001 \\
\hline & $\begin{array}{c}\text { Incidence of irregular vagina } \\
\text { bleeding }\end{array}$ & 15 & 1693 & $0.0 \%$ & 0.995 & $\mathrm{~F}$ & $0.23(0.16,0.31)$ & 0.001 \\
\hline & 6-month relapse rate & 3 & 576 & $0.0 \%$ & 0.683 & $\mathrm{~F}$ & $0.15(0.07,0.35)$ & 0.001 \\
\hline
\end{tabular}

FKQJP, Fuke Qianjin tablet; R, random-effect model; F, fix-effect model. 


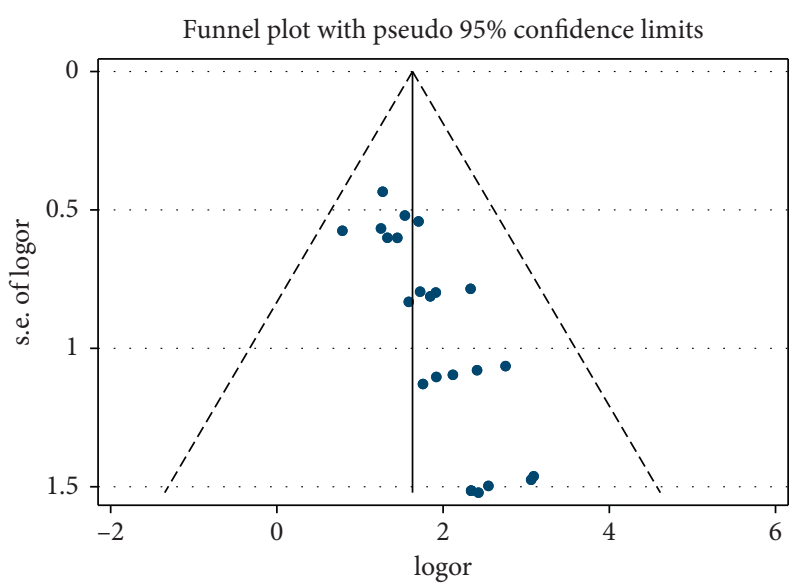

(a)

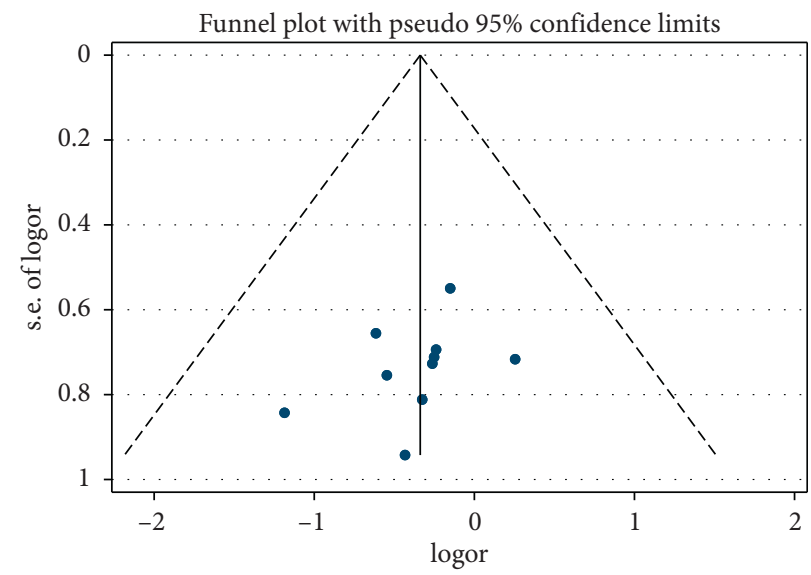

(b)

FIGURE 4: (a) Funnel plot of acute pelvic inflammatory disease of total effective rate. (b) Funnel plot of acute pelvic inflammatory disease of incidence of adverse reaction.

3.4.3. Publication Bias. Perform the publication bias test on total effective rate, endometrial thickness, menstrual normalization rate, and the incidence of irregular vaginal bleeding, and the inverted funnel diagram (Figures 9(a)9(c)) was drawn, respectively. The results of the Harbord test showed that there were no significant publication bias and small sample effect in the total effective rate $(t=0.17$, $p=0.859)$, endometrial thickness $(t=2.04, p=0.060)$, menstrual normalization $(t=-0.90, p=0.39)$, and the incidence of irregular vaginal bleeding $(t=0.17, p=0.867)$. The inverted funnel plots of the 3 outcomes were substantially symmetric.

\section{Discussion}

In recent years, some researchers have evaluated Fuke Qianjin tablets in the treatment of acute pelvic inflammation, chronic pelvic inflammation, and endometritis $[17,152-154]$. It is considered that the clinical effect of Fuke Qianjin tablets combined with antibiotics is better than that of antibiotics alone, which is consistent with the conclusion of this study. The purpose of this study was to compare the efficacy and safety of gynecological Qianjin tablets combined with routine treatment in patients with pelvic inflammatory diseases. The results of this study showed that Fuke Qianjin tablets combined with conventional treatment have better efficacy outcomes for acute pelvic inflammatory disease, chronic pelvic inflammatory disease, and endometritis when compared to using antibiotics alone. Moreover, it can shorten the time of clinical symptom relief, reduce serum inflammatory factor concentration, improve endometrial thickness, improve menstruation, and reduce relapse rate. According to studies reports, the combined application of Fuke Qianjin tablets has no significant adverse reactions, suggesting a strong safety profile.

The occurrence of pelvic inflammatory disease is not only related to a single bacterium. Studies have shown that $30-40 \%$ of pelvic inflammatory cases are caused by multibacterial infection [155]. Therefore, it is necessary to provide broad- spectrum coverage of possible pathogens during treatment. The results of the subgroup analysis showed that the efficacy of Fuke Qianjin tablets combined with antibiotics was not affected by antibiotic types. It demonstrated that Fuke Qianjin tablets are effective in the treatment of a variety of bacteria and have the therapeutic effect of broad-spectrum coverage. Evidence from the PEACH research suggests that short-term and long-term outcomes of oral and intravenous regimens are similar [156], so there is no subgroup analysis of antibiotic administration routes and courses in this study. In addition, two studies of Fuke Qianjin tablets compared with antibiotics (penicillin + metronidazole) showed that Fuke Qianjin tablet was more effective in the treatment of chronic pelvic inflammation. Due to the small number of literature included, more clinical studies need to be carried out to verify the clinical efficacy of Fuke Qianjin tablets alone.

Traditional Chinese medicine has multiple components, and its complexity means it has many potential targets. This makes it more difficult for bacteria to become resistant to it, which has a definite clinical advantage for PID. Fuke Qianjin tablet is extracted from herbs including Suberect spatholobus stem, Jin Ying Gen, Angelica sinensis, and Andrographis paniculata Nees. Suberect spatholobus stem has the effect of relaxing muscles and activating blood circulation; Jin Ying Gen has the effect of fixing essence and astringent intestines; Angelica sinensis has the effect of tonifying blood, harmonizing blood, regulating menstruation, and stopping bleeding; Andrographis paniculata Nees has the effect of cooling blood and detumescence, clearing heat, and detoxification. Related animal experiments and modern pharmacological studies [157-161] showed that Fuke Qianjin tablets may achieve the anti-inflammatory effect by regulating the concentration of serum TNF- $\alpha$, NF-kB, IL-2, IL- 6 , and other factors to reduce the inflammatory response mediated by cellular inflammatory factors. It can also promote the production of $\operatorname{IgA}, \operatorname{IgG}$, and IgM [162], improve the body's immunity, play antibacterial and anti-inflammatory effects, and also improve the body's ability to resist infection. This demonstrated that in the future, due to the increasing resistance of pathogens to antibiotics, 


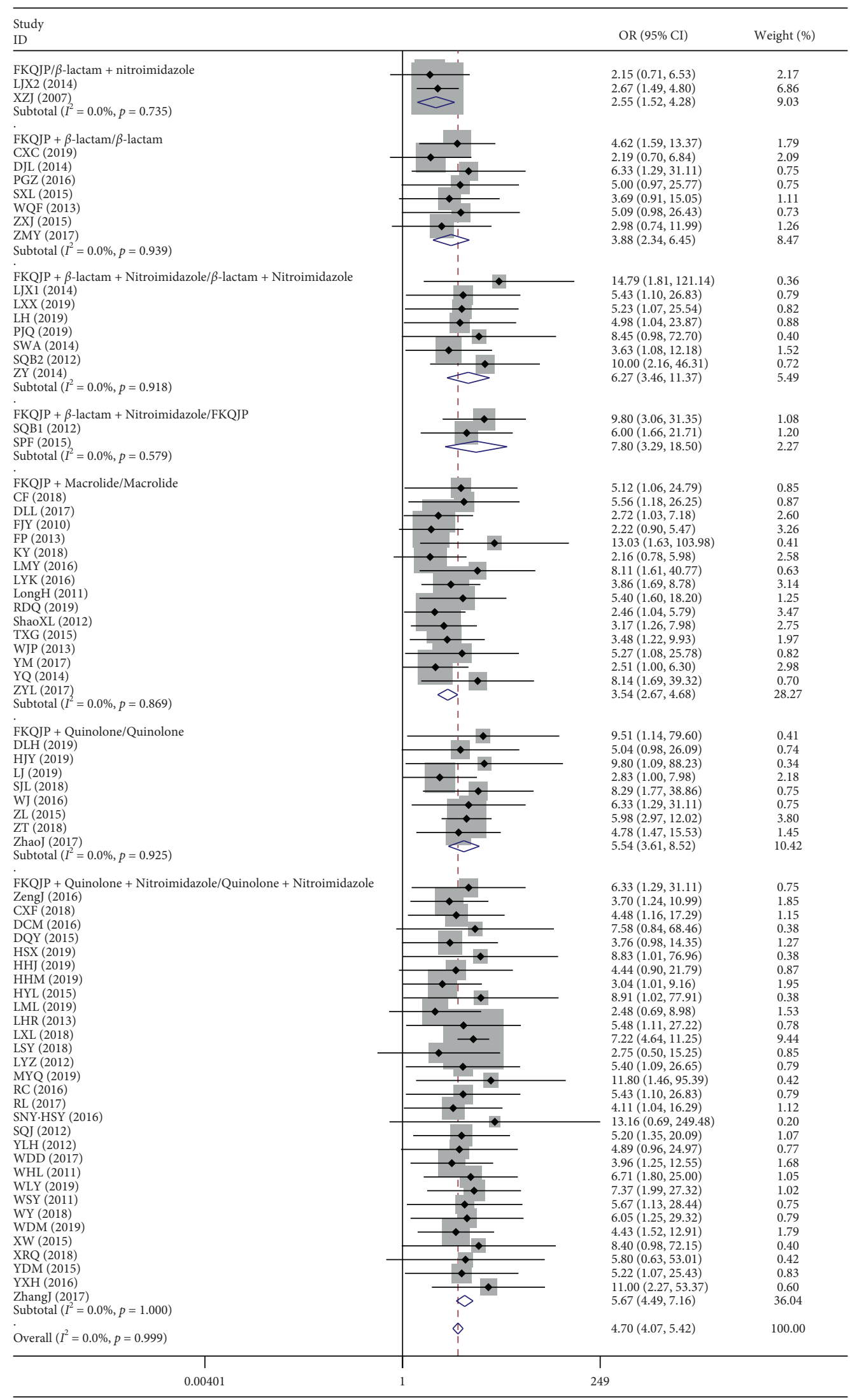

FIGURE 5: Meta-analysis of total effective rate of chronic pelvic inflammatory disease. 


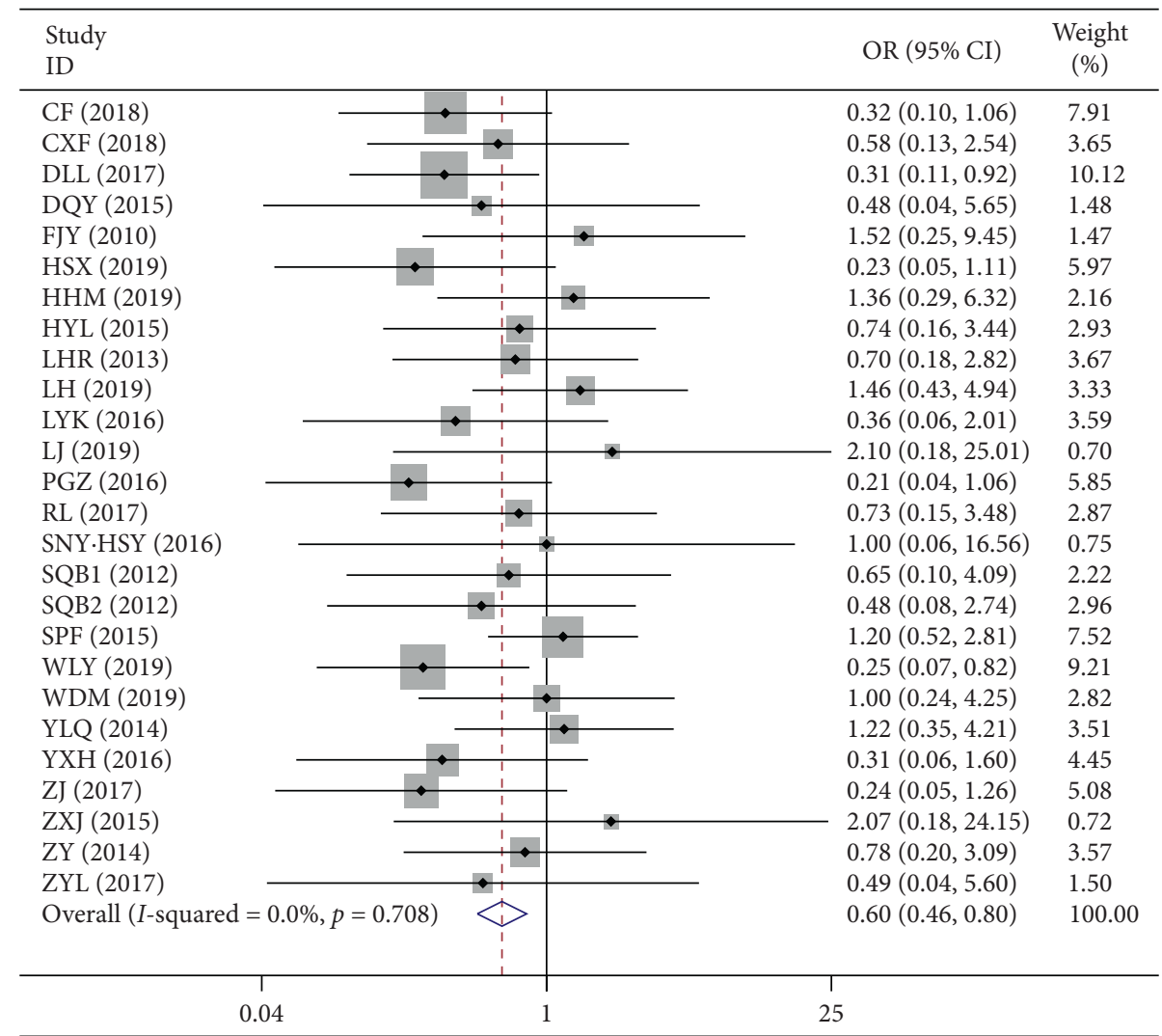

FIgURE 6: Meta-analysis of adverse reaction of chronic pelvic inflammatory disease.

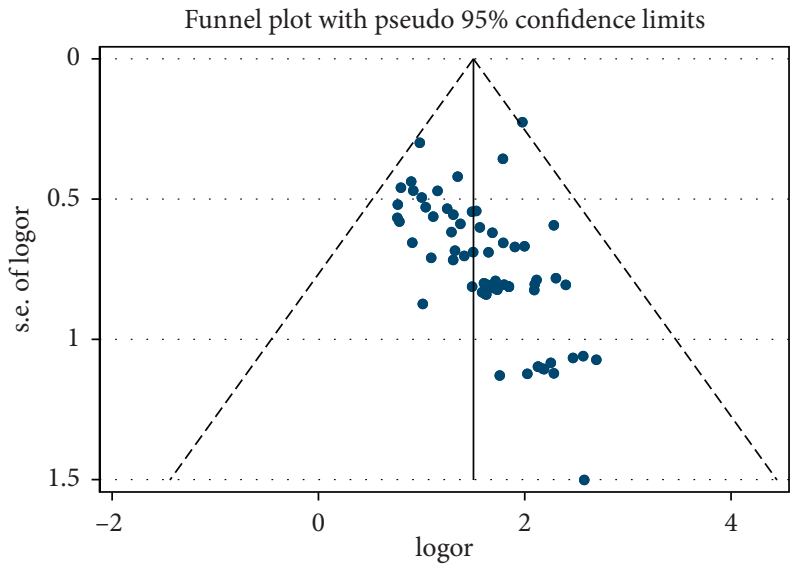

(a)

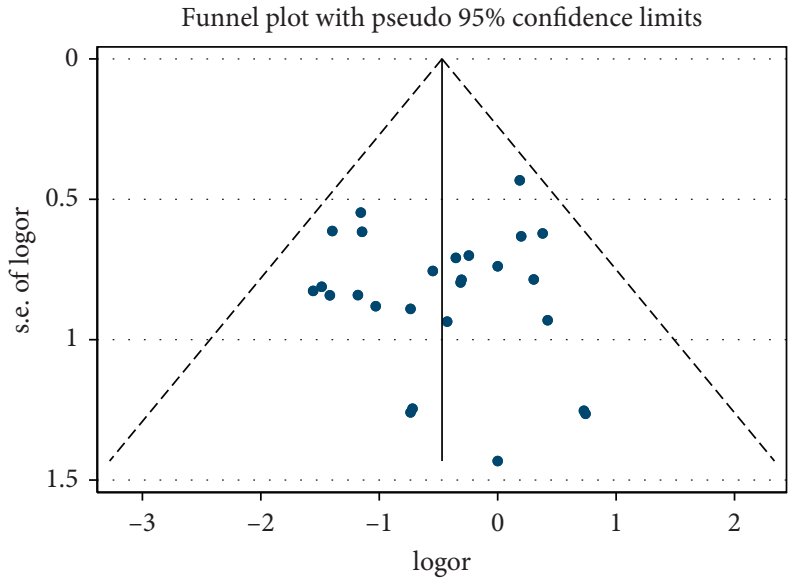

(b)

FIGURE 7: (a) Funnel plot of chronic pelvic inflammatory disease of the total effective rate. (b) Funnel plot of chronic pelvic inflammatory disease of incidence of adverse reaction.

traditional Chinese medicine may play a more important role in the treatment of PID anti-inflammatory. Therefore, it is necessary to carry out a clinical study on the graded dose response of Fuke Qianjin tablets combined with antibiotics to explore whether the combined application can reduce the dose and frequency of antibiotics, so as to alleviate the situation of reduced efficacy due to increased antibiotic resistance.
Heterogeneity between studies is a key issue in metaanalysis. The existence of heterogeneity has an impact on the merger of research results and also directly affects the interpretation of meta-analysis results. This research conducted a subgroup analysis and sensitivity analysis on the outcomes with high heterogeneity. Through a reexamination of studies with high heterogeneity and comparison with other studies, the stability of the sensitive 


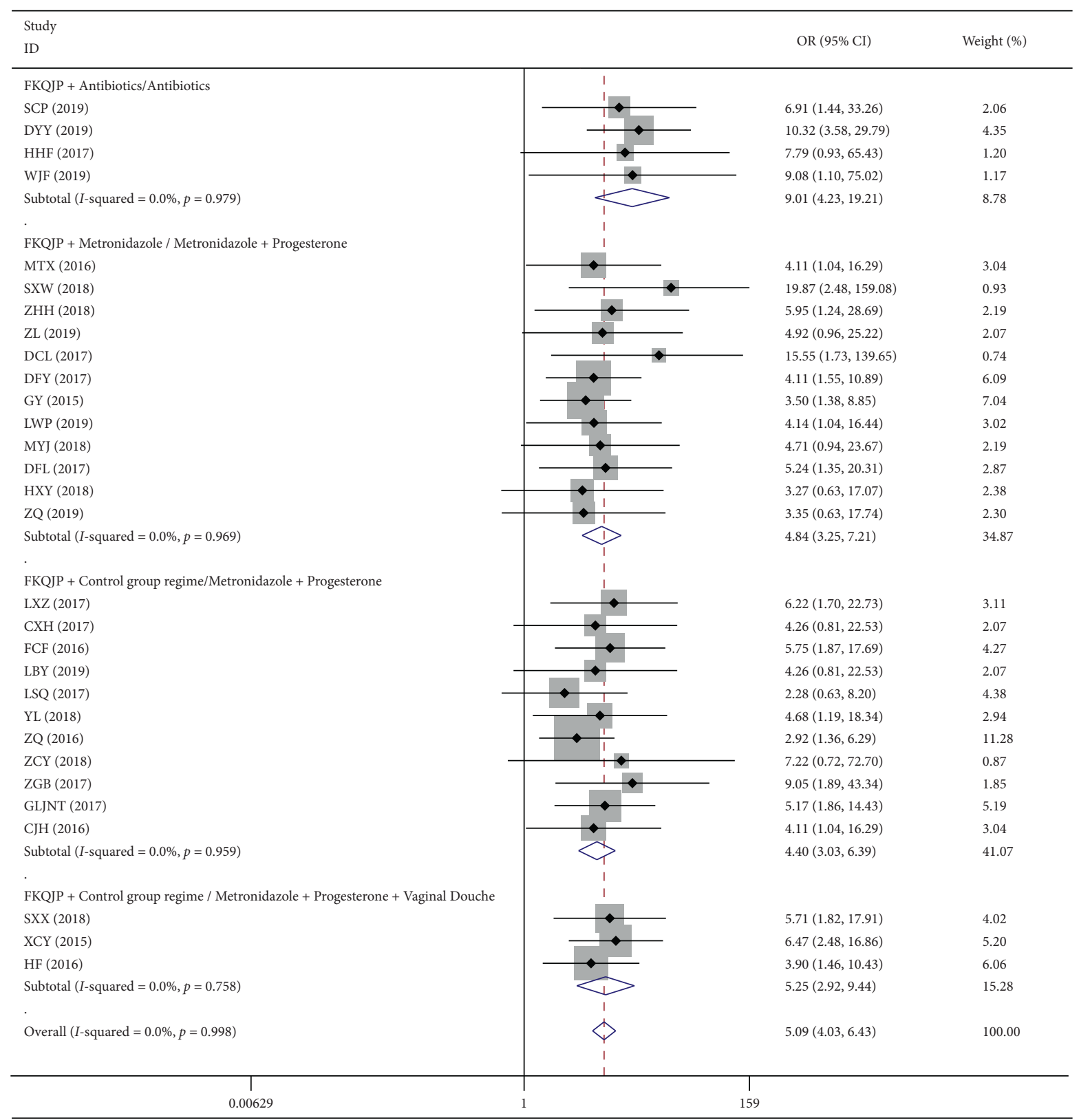

Figure 8: Meta-analysis of total effective rate of endometritis disease.

analysis results to determine the credibility of the research results and whether to retain the studies. In the meta-analysis of acute pelvic inflammatory disease, there was significant heterogeneity in some secondary outcome indicators, which was mainly caused by Zhao and Huang [29] and Fan [38]. The reverification of the two articles showed that the literature quality evaluation was moderately biased risk. After excluding the literature data, the result of meta-analysis was still significant, and the sensitivity analysis showed that the result was stable. In the meta-analysis of chronic pelvic inflammation, two secondary outcome indicators of the remission time of uterine pain and remission time of abdominal pain carried out subgroup analysis according to the course of treatment. The results showed that the heterogeneity significantly decreased, and the difference between the experimental group and the control group was still statistically significant. It can be considered that the results of meta-analysis of these two indicators were robust. Sensitivity analysis was performed on the two secondary outcome indicators of CRP and TNF- $\alpha$ concentration. The outcome indicator of TNF- $\alpha$ concentration has passed the sensitivity analysis, and the meta-analysis results of this indicator can be considered robust. The outcome indicator of CRP concentration in chronic pelvic inflammatory disease cannot pass the sensitivity analysis. It is considered that the reliability of the result of meta-analysis of this indicator was low, and the source of its heterogeneity needs to be further discussed.

This research still has certain limitations. First of all, affected by the quality of the original literature, only 2 articles of the original literature reported the specific 


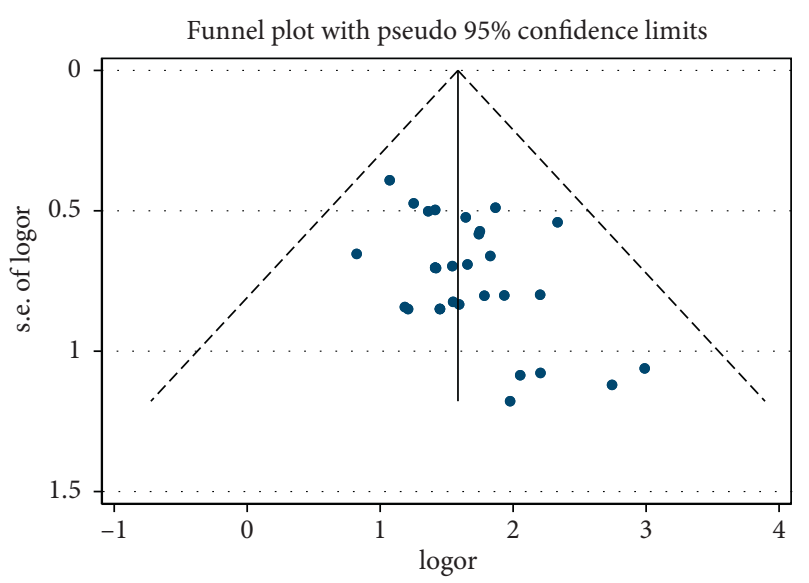

(a)

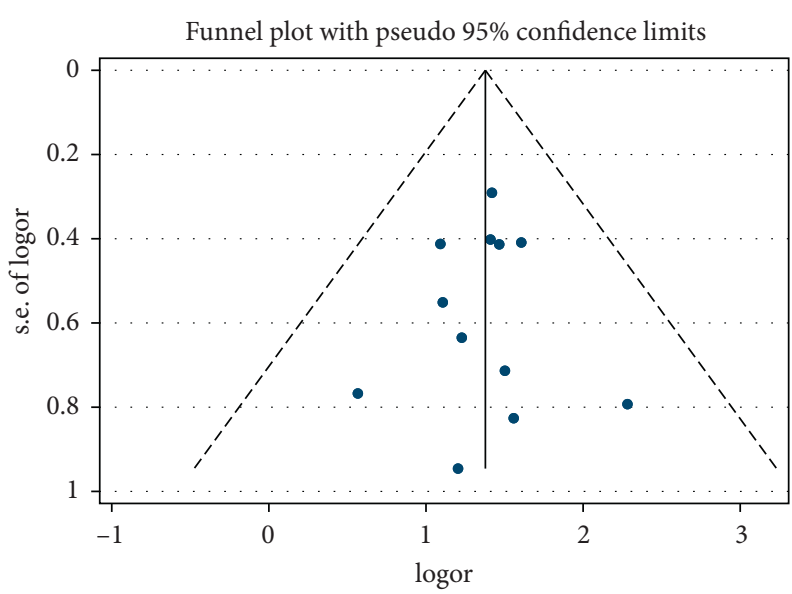

(b)

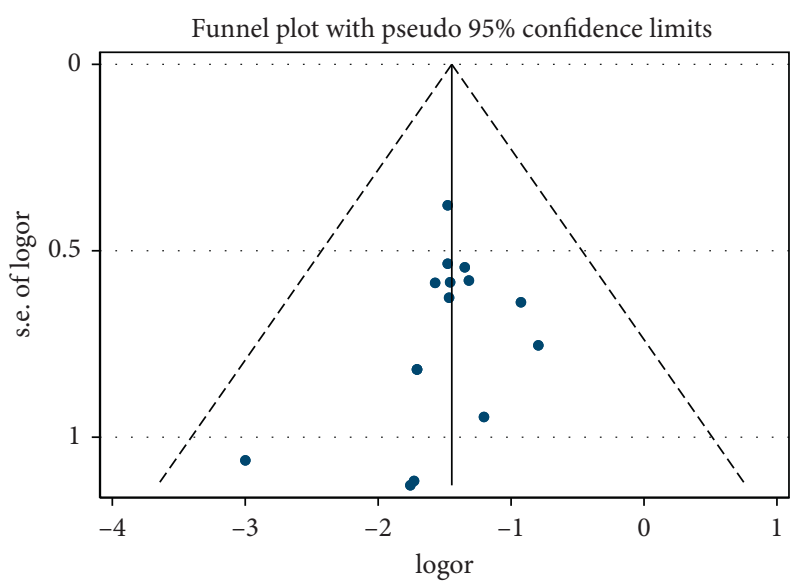

(c)

Figure 9: (a) Funnel plot of endometritis of the total effective rate. (b) Funnel plot of endometritis of menstruation recovery rate. (c) Funnel plot of endometritis of incidence of irregular vaginal bleeding.

implementation of the blind method, and most of them did not report the specific implementation of the random method and the blind method, which may have some potential bias. Second, the subgroup analysis and sensitivity analysis of the secondary outcome indicators with heterogeneity showed that the results were robust. But the number of secondary outcome indicators reported in articles was very small, thereby potentially affecting the strength of the results. Finally, the clinical research of gynecological Fuke Qianjin tablets is only carried out across hospitals in China. The extrapolation of the results on an international scale maybe limited. In order to overcome the above limitations and verify the results of this study, additional high-quality randomized controlled trials that employ a larger sample size are required.

\section{Conclusion}

In order to systematically evaluate the efficacy of Fuke Qianjin tablets in the treatment of pelvic inflammatory diseases and endometritis, this study uses indicators such as total effective rate and incidence of adverse reactions to compare with the combined application of various conventional treatment programs. Results from 125 studies have been observed in the treatment of acute pelvic inflammatory disease, chronic pelvic inflammatory disease, and endometritis. Overall, these studies report positive effects of Fuke Qianjin tablets as adjuvant medication. In addition, the incidence of adverse reactions was not statistically different in the analyzed studies.

\section{Abbreviations \\ CNKI: China National Knowledge Infrastructure \\ VIP: $\quad$ Chinese Scientific Journals Database \\ PID: Pelvic inflammatory disease \\ FKQJP: Fuke Qianjin tablet.}

\section{Data Availability}

The data generated or analyzed during this study are included within this published article. The data used to support the findings of this study have been deposited in the 7 electronic databases, including PubMed, The Cochrane Library, Web of science, CNKI, Wan Fang, VIP, and Sinomed. 


\section{Conflicts of Interest}

The authors declare that they have no conflicts of interest.

\section{Authors' Contributions}

PJ was in charge of the main execution of the research and wrote the main part of the study. All authors contributed to data analysis and drafted and revised the article. Wentao supervised and instructed the study. All authors read and approved the final manuscript.

\section{Acknowledgments}

This work was supported by Zhuzhou Qianjin Pharmaceutical Co., Ltd.

\section{References}

[1] R. L. Sweet, "Treatment of acute pelvic inflammatory disease," Infectious Diseases in Obstetrics and Gynecology, vol. 2011, Article ID 561909, 13 pages, 2011.

[2] D. E. Soper, "Pelvic inflammatory disease," Obstetrics \& Gynecology, vol. 116, no. 2, Part 1, pp. 419-428, 2010.

[3] W.-j. Zhao and G. Q. Huang, "Progress on clinical study of acupuncture treatment for chronic pelvic inflammation," Journal of Acupuncture and Tuina Science, vol. 6, no. 4, pp. 251-256, 2008.

[4] R. C. Brunham, S. L. Gottlieb, and J. Paavonen, "Pelvic inflammatory disease," New England Journal of Medicine, vol. 372, no. 21, pp. 2039-2048, 2015.

[5] Y. Cheng, Y. Yuan, Y. Jin et al., "Acupuncture for chronic pelvic inflammatory disease: a systematic review protocol," Medicine, vol. 97, no. 13, 2018.

[6] C. C. Huang, C. C. Huang, S. Y. Lin et al., "Association of pelvic inflammatory disease (PID) with ectopic pregnancy and preterm labor in Taiwan: a nationwide population-based retrospective cohort study," PLoS One, vol. 14, no. 8, 2019.

[7] I. Sirota, S. Zarek, J. Segars et al., "Potential influence of the microbiome on infertility and assisted reproductive technology," Seminars in Reproductive Medicine, vol. 32, no. 1, pp. 35-42, 2014.

[8] N. Yoshii, T. Hamatani, N. Inagaki et al., "Successful implantation after reducing matrix metalloproteinase activity in the uterine cavity," Reproductive Biology and Endocrinology, vol. 11, no. 1, p. 37, 2013.

[9] V. A. Kushnir, S. Solouki, T. Sarig-Meth et al., "Systemic inflammation and autoimmunity in women with chronic endometritis," American Journal of Reproductive Immunology, vol. 75, no. 6, pp. 672-677, 2016.

[10] M. Trent, H. P. Lehmann, Q. Qian, C. B. Thompson, J. M. Ellen, and K. D. Frick, "Adolescent and parental utilities for the health states associated with pelvic inflammatory disease," Sexually Transmitted Infections, vol. 87, no. 7, pp. 583-587, 2011.

[11] K. Owusu-Edusei, H. W. Chesson, T. L. Gift et al., "The estimated direct medical cost of selected sexually transmitted infections in the United States, 2008," Sexually Transmitted Diseases, vol. 40, no. 3, p. 197, 2013.

[12] Armed Forces Health Surveillance Center (AFHSC), "Acute pelvic inflammatory disease, active component," U.S. $M S M R$, vol. 19, pp. 11-13, 2012.
[13] K. A. Workowski and G. Bolan, "Sexually transmitted diseases treatment guidelines, 2015," Morbidity and Mortality Weekly Report, vol. 64, no. 33, pp. 1-137, 2015.

[14] S. P. Lou and Z. H. Wen, "Clinical practice guidelines for the treatment of pelvic inflammatory diseases with single use/ combined antibiotics in traditional Chinese medicine," in Proceedings of the 19th Annual Meeting of the Chinese Association for Science and Technology-12 Standards Leading the Summit Forum on Academic Innovation and Development of Traditional Chinese Medicine, Changchun, Jilin, China, October 2017.

[15] World Health Organization, Sexually Transmitted Infections, WHO, Geneva, Switzerland, 2019, https://www.who.int/ news-room/fact-sheets/detail/sexually-transmittedinfections-(stis).

[16] Infectious Diseases Cooperation Group of the Chinese Medical Association Obstetrics and Gynecology Branch, "Guidelines for the diagnosis and treatment of pelvic inflammatory diseases (2019 revised edition)," Chinese Journal of Obstetrics and Gynecology, vol. 54, no. 7, pp. 433-437, 2019.

[17] G. X. Li and Q. Zhao, "A systematic review of Fuke Qianjin Capsule (Fuke Qianjin Tablet) combined with antibiotics in the treatment of acute and chronic pelvic inflammatory disease," Journal of Changzhi Medical College, vol. 32, no. 3, pp. 201-207, 2018.

[18] J. P. T. Higgins, D. G. Altman, P. C. Gotzsche et al., "The Cochrane collaboration's tool for assessing risk of bias in randomised trials," British Medical Journal, vol. 343, p. d5928, 2011.

[19] J. P. T. Higgins and S. G. Thompson, "Quantifying heterogeneity in a meta-analysis," Statistics in Medicine, vol. 21, no. 11, pp. 1539-1558, 2002.

[20] J. P. T. Higgins, S. G. Thompson, J. J. Deeks et al., "Measuring inconsistency in meta-analyses," British Medical Journal, vol. 327, no. 7414, pp. 557-560, 2003.

[21] R. DerSimonian and N. Laird, "Meta-analysis in clinical trials," Controlled Clinical Trials, vol. 7, no. 3, pp. 177-188, 1986.

[22] J. Y. Wang and J. X. Zhai, "Stock identity," Encyclopedia of Marine Mammals, vol. 9, no. 10, pp. 1115-1118, 2009.

[23] T. S. Zhang and W. Z. Zhong, "Drawing funnel plot and testing for funnel plot asymmetry in stata," Journal of Evidence Based Medicine, vol. 9, no. 2, pp. 110-114, 2009.

[24] M. Kicinski, D. A. Springate, and E. Kontopantelis, "Publication bias in meta-analyses from the Cochrane database of systematic reviews," Statistics in Medicine, vol. 34, no. 20, pp. 2781-2793, 2015.

[25] R. M. Harbord, M. Egger, and J. A. C. Sterne, "A modified test for small-study effects in meta-analyses of controlled trials with binary endpoints," Statistics in Medicine, vol. 25, no. 20, pp. 3443-3457, 2006.

[26] T. S. Zhang and W. Z. Zhong, "Performance of the nonparametric trim and fill method in stata," Journal of Evidence Based Medicine, vol. 9, no. 4, pp. 240-242, 2009.

[27] C. J. Chen, "Clinical analysis on penicillin combined with Gynecologic Qianjin tablets in treatment of acute pelvic inflammatory," Bao Jian Wen Hui, vol. 6, p. 46, 2018.

[28] M. L. Yan, "Clinical observation on treatment of acute pelvic inflammation with Fuke qianjin tablets combined with antibiotics," Road to Health Magazines, vol. 12, no. 11, pp. 119-120, 2013.

[29] J. Zhao and X. S. Huang, "To explore the clinical effect of Fuke Qianjin tablets combined with penicillin in the treatment of acute pelvic inflammatory disease," Health Guide, vol. 42, p. 50, 2017. 
[30] Y. Dong, "Clinical analysis on penicillin combined with Gynecologic Qianjin Tablets in treatment of acute pelvic inflammatory," Occupation and Health, vol. 30, no. 11, pp. 1576-1578, 2014.

[31] L. Sun and Y. Gao, "Clinical study of Gynecological Qianjin tablets combined with antibiotics in the treatment of Acute pelvic inflammation," Chinese Journal of Trauma and Disability Medicine, vol. 23, no. 22, pp. 75-76, 2015.

[32] S. Q. Tian, "Clinical analysis on penicillin combined with Gynecologic Qianjin Tablets in treatment of acute pelvic inflammatory," China Health Care \& Nutrition, vol. 29, no. 10, p. 61, 2019.

[33] Q. X. Xiong, "Clinical observation on treatment of acute pelvic inflammation with Fuke qianjin tablets combined with antibiotics," Health Care Today, vol. 4, p. 132, 2014.

[34] F. Y. Yu and L. J. Zhou, "Clinical observation on treatment of acute pelvic inflammation with Fuke qianjin tablets combined with antibiotics," Journal of Emerging Traditional Chinese Medicine, vol. 22, no. 6, pp. 992-993, 2013.

[35] S. Rao and Y. M. Yan, "Clinical observation on treatment of acute pelvic inflammation with Fuke qianjin tablets combined with antibiotics," Chinese Journal of Clinical Rational Drug Use, vol. 5, no. 7, pp. 62-63, 2012.

[36] F. M. Sun, "Observation on clinical curative effect of acute pelvic inflammatory in 132 cases," China \& Foreign Medical Treatment, vol. 34, no. 25, pp. 40-41, 2015.

[37] Y. Zhu and J. Li, "Effect of Gynecological Qianjin tablet on Serum CRP, IL-6 and TNF- $\alpha$ levels in patients with Acute pelvic inflammation," Journal of Radioimmunology, vol. 26, no. 2, pp. 182-183, 2013

[38] X. H. Fan, "Analysis of the effectiveness of Gynecological Qianjin tablets combined with antibiotics in the treatment of acute pelvic inflammation," Medical Diet and Health, vol. 5, pp. 31-32, 2018.

[39] Y. Q. Shi, "Observation on the effect of combined application of gynecological Qianjin tablets and antibiotics in the treatment of acute pelvic inflammation," Contemporary Medical Symposium, vol. 13, no. 15, p. 195, 2015.

[40] H. Y. Zang, "Observation on the efficacy of gynecological Qianjin tablets combined with roxithromycin in the treatment of 60 cases of acute pelvic inflammation," Chinese Journal of Modern Drug Application, vol. 6, no. 15, pp. 83-84, 2012.

[41] Y. Z. Zhang, "Observation on the efficacy of gynecological Qianjin tablets combined with oral antibiotics in the treatment of acute pelvic inflammation," Chinese Journal of Modern Drug Application, vol. 6, no. 3, pp. 60-61, 2012.

[42] C. Q. Yu, "Analysis of the effect of moxifloxacin combined with gynecological Qianjin tablets in the treatment of acute pelvic inflammation," Contemporary Medicine Forum, vol. 15, no. 4, pp. 103-104, 2017.

[43] W. R. Lin and W. Wang, "Clinical study of gynecological Qianjin tablets in the treatment of 86 cases of acute pelvic inflammation," Liaoning Journal of Traditional Chinese Medicine, vol. 40, no. 10, pp. 2065-2067, 2013.

[44] G. J. Wang, "Clinical observation of gynecological Qianjin tablets combined with moxifloxacin in the treatment of acute pelvic inflammation," Chinese Journal of Aesthetic Medicine, vol. 20, no. z6, p. 378, 2011.

[45] R. M. Huang and C. Z. Wei, "Clinical analysis of gynecological Qianjin tablets combined with moxifloxacin in the treatment of 60 cases of acute pelvic inflammation," Lingnan Journal of Emergency Medicine, vol. 18, no. 2, pp. 117-118, 2013.
[46] S. Y. Guan, "Clinical effect of gynecological Qianjin tablets combined with antibiotics in the treatment of acute pelvic inflammation," Healthmust-Readmagazine, vol. 11, no. 11, p. 287, 2012.

[47] Y. H. Zhang, "Evaluation of the efficacy of gatifloxacin, metronidazole and gynecological Qianjin tablets in the treatment of acute pelvic inflammation," Contemporary Medicine Forum, vol. 14, no. 13, pp. 105-106, 2016.

[48] N. N. Zhong, "Analysis of the effect of gynecological Qianjin tablets combined with antibiotics in the treatment of acute pelvic inflammation," Chinese Manipulation \& Rehabilitation Medicine, vol. 3, no. 9, pp. 88-89, 2012.

[49] J. Li and Q. Zeng, "Influence of Fuke qianjin tablets on expression of serum inflammatory mediator in patients with acute pelvic inflammation," Chinese Pharmacy, vol. 23, no. 24, pp. 33-35, 2014.

[50] J. X. Li, "Observation on the therapeutic effect of 120 cases of chronic pelvic inflammation," Contemporary Medicine, vol. 20 , no. 23 , p. $160,2014$.

[51] Z. J. Xiang, "Treatment of 102 cases of chronic pelvic inflammation with gynecological Qianjin tablet," Hubei Journal of Traditional Chinese Medicine, vol. 8, p. 41, 2007.

[52] X. C. Cong, "Observation on the efficacy of antibiotics combined with gynecological Qianjin tablets in the treatment of chronic pelvic inflammation," Health Horizon, vol. 46, no. 10, p. $49,2019$.

[53] J. L. Dai, "Clinical observation of gynecological Qianjin tablets combined with antibiotics in the treatment of chronic pelvic inflammation," Practical Clinical Journal of Integrated Traditional Chinese and Western Medicine, vol. 14, no. 3, p. 55,2014

[54] G. Z. Peng, "Clinical efficacy and safety evaluation of gynecological Qianjin tablets combined with amoxicillin clavulanate potassium tablets in the treatment of chronic pelvic inflammation," For All Health, vol. 10, no. 1, p. 128, 2016.

[55] X. L. Su, "Analysis of the efficacy of antibiotics combined with gynecological Qianjin tablets in the treatment of 64 cases of chronic pelvic inflammation," Journal of Aerospace Medicine, vol. 26, no. 7, pp. 849-850, 2015.

[56] Q. F. Wang and H. R. Ma, "Clinical observation on 70 cases of chronic pelvic inflammation treated by combination of traditional Chinese and Western Medicine," Seek Medical and Ask the Medicine, vol. 11, no. 1, p. 161, 2013.

[57] X. J. Zhang and D. Xu, "Clinical efficacy and safety evaluation of gynecological Qianjin tablets combined with amoxicillin clavulanate potassium tablets in the treatment of chronic pelvic inflammation," Liaoning Journal of Traditional Chinese Medicine, vol. 42, no. 5, pp. 1001-1002, 2015.

[58] M. Y. Zhong, "Clinical evaluation of penicillin combined with gynecological Qianjin tablets in the treatment of pelvic infection in gynecological patients," Anti-Infection Pharmacy, vol. 14, no. 3, pp. 636-638, 2017.

[59] X. X. Li, "Observation on the efficacy of gynecological Qianjin tablets combined with Ceftriaxone Sodium and Metronidazole injection in the treatment of chronic pelvic inflammation," Journal of North Pharmacy, vol. 16, no. 6, pp. 60-61, 2019.

[60] H. Liu and G. Yang, "Observation on the efficacy of gynecological Qianjin tablets combined with Ceftriaxone Sodium and Metronidazole injection in the treatment of chronic pelvic inflammation," Anti-Infection Pharmacy, vol. 16, no. 8, pp. 1440-1442, 2019.

[61] J. Q. Pan, "Observation on the efficacy of gynecological Qianjin tablets combined with Ceftriaxone Sodium and 
Metronidazole injection in the treatment of chronic pelvic inflammation," Health For Everyone, vol. 17, 2019.

[62] W. A. Shao, "Analysis of clinical therapeutic effect of chronic pelvic inflammation," Medical Information, vol. 18, p. 512, 2014.

[63] Q. B. Song, "Analysis of the efficacy of gynecological Qianjin tablets combined with antibiotics in the treatment of chronic pelvic inflammation," Strait Pharmaceutical Journal, vol. 24, no. 6, pp. 158-160, 2012.

[64] Y. Zhang, "Clinical observation on the treatment of 54 cases of chronic pelvic inflammation with gynecological Qianjin tablet," Modern Diagnosis and Treatment, vol. 25, no. 11, pp. 2451-2452, 2014.

[65] P. F. Sun and X. J. Lv, "Analysis of the efficacy of gynecological Qianjin tablets combined with antibiotics in the treatment of chronic pelvic inflammation," Modern Women, vol. 6, p. 40, 2015.

[66] F. Chen, "The clinical effect and safety analysis of clindamycin combined with Fuke Qianjin tablets in the treatment of chronic pelvic inflammatory disease," Health Horizon, vol. 14, pp. 41-42, 2018.

[67] L. Deng, "The clinical effect and safety analysis of clindamycin combined with Fuke Qianjin tablets in the treatment of chronic pelvic inflammatory disease," China Modern Medicine, vol. 24, no. 35, pp. 128-130, 2017.

[68] J. Fang and L. Li, "Clindamycin phosphate tablets combined with Fuke qianjin tablets in treating 63 cases of chronic pelvic inflammation," China Pharmaceuticals, vol. 19, no. 9, p. 75, 2010.

[69] P. Fu, "Clinical observation on treatment of chronic pelvic inflammation with Fuke qianjin tablets combined with clindamycin phosphate," Journal of Liaoning University of Traditional Chinese Medicine, vol. 15, no. 8, pp. 204-205, 2013.

[70] Y. Kang, "Observation on the effect of Fuke Qianjin tablet on the sequelae of pelvic inflammatory disease," Women's Health Research, vol. 20, pp. 64-181, 2018.

[71] M. Li, "Clinical effect of Fuke qianjin tablets combined with clindamycin phosphate in the treatment of chronic pelvic inflammation," World Latest Medicine Information, vol. 16, no. 96, p. 173, 2016.

[72] Y. Liu, "Analysis of efficacy and safety of Fuke qianjin tablets combined with western medicine in the treatment of chronic pelvic inflammation," Public Medicinal Forum Magazine, vol. 20, no. 3, pp. 354-355, 2016.

[73] H. Long, "Analysis of the therapeutic effect of Fuke Qianjin tablets combined with antibiotics on 180 cases of chronic pelvic inflammation," Guide of China Medcine, vol. 9, no. 16, p. 146, 2011.

[74] D. Ran, "Clindamycin-assisted Fuke Qianjin tablets in the treatment of chronic pelvic inflammatory disease: clinical efficacy and impact analysis," Mother and Infant World, vol. 1, p. 124, 2019.

[75] X. Shao and Q. Wang, "Clinical observation on treatment of 87 cases of chronic pelvic inflammation with Fuke Qianjin tablets combined with clindamycin phosphate," Hebei Journal of Traditional Chinese Medicine, vol. 34, no. 11, pp. 1658-1660, 2012.

[76] X. Tian, "Clindamycin phosphate tablets and Fuke qianjin tablets combined treatment of pelvic inflammation," Medicine, vol. 3, 2015.

[77] J. Wu, "Clindamycin assisted Fuke Qianjin tablets in the treatment of 100 cases of chronic pelvic inflammatory disease," Journal of North Pharmacy, vol. 10, no. 6, pp. 49-50, 2013.
[78] M. Yan, "Clindamycin assisted Fuke Qianjin Tablets in the treatment of chronic pelvic inflammatory disease," China Health Care Nutrition, vol. 27, no. 14, p. 367, 2017.

[79] L. Yu, "Clindamycin phosphate tablets combined with Fuke Qianjin tablets in the treatment of chronic pelvic inflammation," Chinese Journal of Clinical Rational, vol. 7, no. 15, pp. 114-115, 2014.

[80] Y. Zhu, "Clindamycin assisted Fuke Qianjin tablets in the treatment of chronic pelvic inflammatory disease," Journal of Practical Gynecologic Endocrinology, vol. 4, no. 30, pp. 25-27, 2017.

[81] L. Dong, "Clinical effect of Fuke Qianjin tablets combined with Fleroxacin in the treatment of chronic pelvic inflammatory disease," Henan Medical Research, vol. 28, no. 15, pp. 2799-2800, 2019.

[82] J. He, "Clinical effect of Fuke Qianjin tablets combined with Fleroxacin in the treatment of chronic pelvic inflammatory disease," Journal of Practical Gynecologic Endocrinology, vol. 6, no. 29, pp. 36-44, 2019.

[83] J. Lv, "Clinical effect of antibiotics combined with Fuke Qianjin tablets in the treatment of chronic pelvic inflammatory disease," Health Required, vol. 4, p. 61, 2019.

[84] J. Wang, "Fuke qianjin capsule in treating 53 cases of sequelae of pelvic inflammatory disease," World Latest Medicine Information, vol. 16, no. 51, p. 98, 2016.

[85] J. Shang, "The effect of combined use of levofloxacin and Fuke Qianjin tablets on patients with chronic pelvic inflammatory disease and its influence on quality of life," Anti-Infection Pharmacy, vol. 15, no. 1, pp. 160-162, 2018.

[86] L. Zhang and A. Guo, "Clinical evaluation of Fuke qianjin tablets combined with levofloxacin lactate injection in the treatment of chronic pelvic inflammation," Women's Health Research, vol. 22, pp. 201-211, 2015.

[87] T. Zhang and Q. Zhang, "Observation on therapeutic effect of Fuke qianjin tablets combined with antibiotics on 160 cases of chronic pelvic inflammation," Journal of Clinical Research, vol. 35, no. 11, pp. 2171-2172, 2018.

[88] J. Zhao and R. Guo, "Effect of levofloxacin combined with Fuke qianjin tablets on inflammatory factors in patients with chronic pelvic inflammation," China Practical Medicine, vol. 12, no. 5, pp. 113-115, 2017.

[89] J. Zeng, "Analysis of clinical effect of Fuke qianjin tablets on chronic pelvic inflammation," China Foreign Medical Treatment, vol. 35, no. 11, pp. 161-162, 2016.

[90] X. Chen, "Effect of Fuke qianjin tablets combined with antibiotics on chronic pelvic inflammation," Contemporary Medical Symposium, vol. 16, no. 19, pp. 2-4, 2018.

[91] C. Deng, "Observation of clinical efficacy of combined Fuke qianjin tablets and antibiotics in treating chronic pelvic inflammation," Health Way, vol. 9, pp. 123-124, 2016.

[92] Q. Ding, "Treatment of chronic pelvic inflammation with Fuke qianjin tablets combined with antibiotics," World Latest Medicine Information, vol. 15, no. 52, p. 58, 2015.

[93] S. He, "Clinical effect of antibiotics combined with gynecological Qianjin tablets in patients with chronic pelvic inflammatory disease," World Latest Medicine Information, vol. 19, no. 77, pp. 167-169, 2019.

[94] H. Hu and C. Li, "Observation on the clinical effect of antibiotics combined with Fuke Qianjin tablets in the treatment of chronic pelvic inflammation," Clinical Research, vol. 27, no. 3, pp. 121-123, 2019.

[95] H. Huang and G. Chen, "Clinical effect of Fuke Qianjin tablets combined with antibiotics in the treatment of chronic 
pelvic inflammatory disease," Shanghai Medical \& Pharmaceutical Journal, vol. 40, no. 5, pp. 33-35, 2019.

[96] Y. Hang, "Combination of Fuke qianjin tablets and antibiotics in the treatment of 120 cases of chronic pelvic inflammation," Oriental Diet-Therapy and Health Care, vol. 10, p. 29, 2015.

[97] M. L. Le, "Evaluation of the therapeutic effect of antibiotics and Fuke Qianjin tablets in the treatment of chronic pelvic inflammatory disease," Health Horizon, vol. 6, pp. 32-33, 2019.

[98] H. R. Li, "Treatment of 45 cases of chronic pelvic inflammation with Fuke qianjin tablets," Chinese Journal of Modern Distance Education in Traditional Chinese Medicine, vol. 11, no. 21, pp. 29-30, 2013.

[99] X. L. Li, "Effect of Fuke qianjin tablets combined with levofloxacin and metronidazole on chronic pelvic inflammation," Henan Medical Research, vol. 27, no. 16, pp. 2984-2985, 2018.

[100] S. Y. Lin and W. F. Fan, "Effect of Fuke qianjin tablets combined with antibiotics on hemorheology and immune function in patients with chronic pelvic inflammation," World Chinese Medicine, vol. 13, no. 10, pp. 2510-2513, 2018.

[101] Y. Z. Liu, "Observation of therapeutic effect of Fuke qianjin tablets on chronic pelvic inflammation," Chinese Manipulation \& Rehabilitation Medicine(Mid-Term), vol. 3, no. 11, pp. 119-120, 2012.

[102] Y. Q. Ming, "Observation on the effect of antibiotics combined with Fuke Qianjin tablets in the treatment of chronic pelvic inflammation," Journal of Practical Gynecologic Endocrinology, vol. 6, no. 13, p. 109, 2019.

[103] C. Ren, "Analysis of the effect of Fuke Qianjin tablets and antibiotics on chronic pelvic inflammation," Contemporary Medicine Forum, vol. 14, no. 17, p. 144, 2016.

[104] L. Ren, "The effect of antibiotics combined with Fuke Qianjin tablets in the treatment of patients with pelvic inflammatory disease," Medical Equipment, vol. 30, no. 11, pp. 103-104, 2017.

[105] N. Y. H. S. Y. Sha, "Observation on therapeutic effect of Fuke qianjin tablets in the treatment of 45 cases of chronic pelvic inflammation," Medical Information, vol. 29, no. 23, pp. 91-92, 2016.

[106] Q. J. Shen, "Observation of therapeutic effect of Fuke qianjin tablets combined with antibiotics on chronic pelvic inflammation," Guide Of China Medicine, vol. 10, no. 6, pp. 23-25, 2012.

[107] L. H. Tan and Y. H. Chang, "Observation of clinical efficacy of Fuke qianjin tablets combined with antibiotics in treating chronic pelvic inflammation," Progress in Modern Biomedicine, vol. 12, no. 5, pp. 951-953, 2012.

[108] D. D. Wang, "Observation of clinical efficacy of Fuke qianjin tablets combined with antibiotics in treating chronic pelvic inflammation," Modern Diagnosis \& Treatment, vol. 28, no. 14 , pp. $2606-2607,2017$.

[109] H. L. Wang, "Analysis of the therapeutic effect of Fuke Qianjin tablets combined with antibiotics on 180 cases of chronic pelvic inflammation," Jilin Medical Journal, vol. 32, no. 6, p. 1126, 2011.

[110] L. Y. Wang, "Clinical study on treatment of chronic pelvic inflammation with Fuke qianjin tablets and western medicine," Journal of New Chinese Medicine, vol. 51, no. 5, pp. 190-192, 2019.

[111] S. Y. Wang, "Clinical study on treatment of chronic pelvic inflammation with integrated traditional Chinese and western medicine," Journal of Chinese Medicine, vol. 26, no. 09, pp. 1135-1136, 2011.

[112] Y. Wang, "Effect of levofloxacin and Fuke Qianjin tablets on inflammatory cytokines in patients with chronic pelvic inflammation," Modern Health (Second Half Month Edition), vol. 5, pp. 103-104, 2018.

[113] D. Wei and C. Z. Gan, "Observation on the effect of combination of Chinese and western medicine on chronic pelvic inflammation," Journal of Practical Traditional Chinese Medicine, vol. 35, no. 10, pp. 1232-1233, 2019.

[114] W. Xie, "Observation of therapeutic effect of Fuke Qianjin tablets combined with antibiotics on patients with chronic pelvic inflammation," Clinical Journal of Chinese Medicine, vol. 7, no. 8, pp. 48-49, 2015.

[115] R. Q. Xu, "Analysis of the observation report on the therapeutic effect of Fuke Qianjin tablets combined with antibiotics in the treatment of chronic pelvic inflammatory disease," Health Required, vol. 36, p. 221, 2018.

[116] D. M. Yang, "Efficacy analysis of Fuke qianjin tablets combined with antibiotics in treating chronic pelvic inflammation," World Latest Medicine Information, vol. 15, no. 39 , p. 110,2015

[117] X. H. Yu and Y. F. Qiu, "Observation of therapeutic effect of antibiotics combined with Chinese patent medicine on chronic pelvic inflammation," Journal of Hubei College of Traditional Chinese Medicine, vol. 18, no. 2, pp. 72-73, 2016.

[118] J. Zhang, "Clinical observation of Gynecological Qianjin tablet in the treatment of chronic pelvic inflammation," Inner Mongolia Traditional Chinese Medicine, vol. 36, no. 15, p. 63, 2017.

[119] F. F. Shen and A. C. Yuan, "To explore the clinical effect of antibiotics combined with Fuke Qianjin tablets in the treatment of endometritis," Journal of North Pharmacy, vol. 14, no. 10, p. 75, 2017.

[120] C. P. Sun and L. X. Song, "Explore the effectiveness of drugs for endometritis," Journal of Practical Gynecologic Endocrinology, vol. 6, no. 20, p. 43, 2019.

[121] Y. Y. Deng and L. Cheng, "Observation on the clinical effect of antibiotics and Fuke Qianjin tablets in the treatment of endometritis," Journal of Clinical Rational Drug Use, vol. 12, no. 5, pp. 94-95, 2019.

[122] Y. Liu, "Analysis of the clinical effect of antibiotics combined with Fuke Qianjin tablets in the treatment of endometritis," Journal of Practical Gynecologic Endocrinology, vol. 6, no. 28, p. $49,2019$.

[123] H. F. He, "Clinical study on treatment of 60 cases of endometritis with levofloxacin and Fuke qianjin tablets," Clinical Medicine Research and Practice, vol. 2, no. 9, pp. 72-73, 2017.

[124] J. F. Wu and Y. Wang, "The effect of levofloxacin combined with Fuke Qianjin tablets on 120 cases of endometritis," World Latest Medicine Information, vol. 19, no. 84, pp. 142-144, 2019.

[125] T. X. Ma, "The effect of metronidazole and Fuke Qianjin tablets in the treatment of endometritis," Contemporary Medicine Forum, vol. 14, no. 15, pp. 45-46, 2016.

[126] X. W. Sun, "Study on the treatment of endometritis with antibiotics combined with Fuke Qianjin tablets," Diet Health, vol. 5, no. 25, pp. 81-82, 2018.

[127] H. H. ZhangQ. Zhang et al., "Study on the therapeutic effect of antibiotics combined with Fuke Qianjin tablets on endometritis," Chinese Community Physician, vol. 34, no. 3, pp. 88-90, 2018. 
[128] L. Zhang, "Analysis of the effect of Fuke Qianjin tablets combined with metronidazole in the treatment of endometritis," Contemporary Medical Symposium, vol. 17, no. 12, pp. 176-177, 2019.

[129] C. L. Dong, "Analysis of the effect of antibiotics combined with Fuke Qianjin tablets in the treatment of endometritis," The Medical Forum, vol. 21, no. 31, pp. 4427-4428, 2017.

[130] F. Y. Du and L. Y. Zhang, "The clinical effect of antibiotics and Fuke Qianjin tablets in the treatment of endometritis," The Medical Forum, vol. 21, no. 35, pp. 5000-5001, 2017.

[131] Y. Guo, "Analysis of the clinical effect of antibiotics combined with Fuke Qianjin tablets in the treatment of endometritis," Heilongjiang Medical Journal, vol. 39, no. 03, pp. 291-292, 2015.

[132] W. P. Liu, "Observation on the effect of Fuke Qianjin tablets combined with metronidazole in the treatment of endometritis," Regimen, vol. 22, no. 9, p. 205, 2019.

[133] Y. J. Meng, "Study and analysis of the therapeutic effect of antibiotics combined with Fuke Qanjin tablets on endometritis," PSY, vol. 6, pp. 89-90, 2018.

[134] F. L. Du, "Analysis of the therapeutic effect of Fuke Qianjin tablets combined with antibiotics on endometritis," China Health Care \& Nutrition, vol. 27, no. 20, p. 4, 2017.

[135] X. Y. Han, "Analysis of the clinical effect of antibiotics combined with Fuke Qianjin tablets in the treatment of endometritis," Health Way, vol. 4, 2018.

[136] Q. Zheng, "Observation on the effect of antibiotics combined with Fuke Qianjin tablets in the treatment of 80 patients with endometritis," Health Guide, vol. 25, pp. 22-23, 2019.

[137] X. Z. Liu, "Analysis of the clinical effects of metronidazole, medroxyprogesterone and Fuke Qianjin tablets in the treatment of endometritis," The World Clincal Medcine, vol. 11, no. 10, pp. 141-144, 2017.

[138] X. H. Chen, "Clinical effect of antibiotics combined with gynecologic Qianjin tablets on Endometritis," Medical Journal of Chinese People's Health, vol. 29, no. 17, pp. 82-83, 2017.

[139] C. F. Fan, "Study on the clinical effect of antibiotics combined with Fuke Qianjin tablets in the treatment of endometritis," Maternal and Child Health Care of China, vol. 31, no. 15, pp. 3040-3042, 2016.

[140] B. Y. Lin, "The clinical effect of antibiotics combined with hormones and Fuke Qianjin tablets in the treatment of patients with endometritis," Medical Equipment, vol. 32, no. 9, pp. 104-105, 2019.

[141] S. Q. LouP. H. Yang et al., "Analysis of clinical effect of Fuke Qianjin tablets on endometritis," Journal of North Pharmacy, vol. 14, no. 4, pp. 178-179, 2017.

[142] L. Yuan, "Clinical effect of antibiotics combined with Fuke Qianjin tablets in the treatment of endometritis," Chinese and Foreign Medical Research, vol. 16, no. 8, pp. 153-154, 2018.

[143] Q. Zhang, "Application effect of antibiotics combined with Fuke Qianjin tablets in the treatment of endometritis," Women's Health Research, vol. 17, pp. 69-78, 2016.

[144] C. Y. Zhao, "Analysis of the clinical effect of antibiotics combined with Fuke Qianjin tablets in the treatment of endometritis," Special Health, vol. 13, p. 237, 2018.

[145] G. B. Zhang, "Observation on the clinical efficacy of antibiotics combined with Fuke Qianjin tablets in the treatment of endometritis," Chinese Baby, vol. 1, p. 99, 2017.

[146] M. Guriganati and D. Rezimu, "Analysis of the clinical effect of antibiotics combined with Fuke Qianjin tablets in the treatment of endometritis," World Latest Medicine Information, 2017; vol. 17, no. 17, p. 76.

[147] J. H. Chang, "Observation on the therapeutic effect of antibiotics combined with Fuke Qianjin tablets in the treatment of endometritis," Chinese Baby, vol. 15, p. 93, 2016.

[148] A. Rehati and L. H. Zhou, "Clinical effect of antibiotics and Fuke Qianjin tablets in the treatment of endometritis," Guide of China Medicine, vol. 16, no. 23, pp. 164-165, 2018.

[149] P. Zhang, "Analysis of the efficacy of antibiotics and gynecological qianjin tablets in the treatment of endometritis," China \& Foreign Medical Treatment, vol. 37, no. 22, pp. 101-103, 2018.

[150] C. Y. Xie and H. Wang, "Clinical efficacy and mechanism action of antibiotics joint Fuke qianjin pian in treatment of uterus meningitis," Chinese and Foreign Medical Research, vol. 13, no. 23, pp. 48-49, 2015.

[151] F. Hong, "Clinical study on the treatment of endometritis with antibiotics combined with Fuke Qianjin tablets," Electronic Journal of Practical Gynecologic Endocrinology, vol. 3, no. 20, pp. 170-172, 2016.

[152] Y. S. Qin and B. L. Shi, "A systematic review of the efficacy and safety of gynecological Qianjin tablets in the treatment of chronic pelvic inflammation," Journal of Tianjin University Traditional Chinese Medicine, vol. 37, no. 5, pp. 427-432, 2018.

[153] L. Wang and R. Xiu, "Meta-analysis on efficacy of Fuke Qianjin tablets (capsules) combined with antibiotics in treatment of endometritis," China Journal of Chinese Materia Medical, vol. 41, no. 16, pp. 3090-3095, 2016.

[154] X. H. Chen, "Meta analysis of gynecological Qianjin tablets in the treatment of chronic pelvic inflammation," Journal of New Chinese Medicine, vol. 46, no. 11, pp. 216-217, 2014.

[155] W. M. McCormac, "Pelvic inflammatory disease," The New England Journal of Medicine, vol. 330, pp. 115-119, 1994.

[156] R. B. Ness, D. E. Soper, R. L. Holley et al., "Effectiveness of inpatient and outpatient treatment strategies for women with pelvic inflammatory disease: results from the pelvic inflammatory disease evaluation and clinical health (peach) randomized trial," American Journal of Obstetrics and Gynecology, vol. 186, no. 5, pp. 929-937, 2002.

[157] X. Li, "Effect of Fuke qianjin tablet on regulation of inflammatory cytokines in uterus tissue and endometrial cell apoptosis," in Chronic Pelvic Inflammatory Disease of Rats DHunan University of Chinese Medicine, Changsha, China, 2013.

[158] Y. B. Lu and J. Y. Qu, "Effect of Fuke Qianjin tablet on TNF- $\alpha$ and IL2 mRNA transcription in the uteri and ovary tissues of acute pelvic inflammatory disease in rats," Chinese Traditional Patent Medicine, vol. 34, no. 1, 2012.

[159] J. Nie, Effect of Fuke Qianjin Tablets on Fas/Fas1 and NF- $\kappa B /$ I $\kappa$ B Signal Pathway in Ovary and Fallopian Tube Tissues of Rats with Chronic Pelvic Inflammation D, Hunan University of Chinese Medicine, Changsha, China, 2014.

[160] J. L. YuanJ. S. Guo et al., "The influence of gynecologic qianjin tablet to the content of TNF- $\alpha$, NF- $\kappa$ B in serum in rats pelvic inflammatory disease model," Chinese Medicne Modern Distance Education of China, vol. 12, no. 7, pp. 150-151, 2014.

[161] Z. Y. Shi and J. S. Guo, "Influence of Fuke Qianjin tablets on NF- $\kappa$ B, IL6 mRNA in uterine and ovary tissues of acute pelvic inflammatory models of rats," Chinese Pharmacological Bulletin, vol. 27, no. 12, 2011.

[162] J. L. Yuan and J. S. Guo, "Effect of Qianjin tablets on serum IgA, IgG and IgM in acute pelvic inflammation in rats," Journal of TCM Univ. of Hunan, vol. 30, no. 9, 2010. 